\title{
Salt-Induced Thermochromism of a Conjugated Polyelectrolyte
}

\author{
Lisa Peterhans ${ }^{1}$, Elisa Alloa ${ }^{2}$, Yauhen Sheima ${ }^{1}$, Laurent Vannay ${ }^{3}$, Mario Leclerc ${ }^{4}$, Clémence
}

Corminboeuf $^{3}$, Sophia C. Hayes ${ }^{2 *}$ and Natalie Banerji ${ }^{1,5 *}$

\begin{abstract}
${ }^{1}$ Department of Chemistry, University of Fribourg, Chemin du Musée 9, CH-1700 Fribourg, Switzerland
${ }^{2}$ Department of Chemistry, University of Cyprus, P.O. Box 20537, 1678, Nicosia, Cyprus

${ }^{3}$ Laboratory for Computational Molecular Design, Institute of Chemical Sciences and Engineering, Ecole

Polytechnique Fédérale de Lausanne (EPFL), CH-1015 Lausanne, Switzerland

${ }^{4}$ Department of Chemistry, Université Laval, G1K 7P4 Quebec City, Quebec, Canada.
\end{abstract}

${ }^{5}$ Department of Chemistry and Biochemistry, University of Bern, Freiestrasse 3, CH-3012 Bern, Switzerland

\begin{abstract}
We report here the photophysical properties of a water-soluble conjugated polythiophene with cationic side-chains. When dissolved in aqueous buffer solution (PBS, phosphate buffered saline), there is ordering of the polymer chains due to the presence of the salts, in contrast to pure water, where a random-coil conformation is adopted at room temperature. The ordering leads to a pronounced colour change of the solution (the absorption maximum shifts from $400 \mathrm{~nm}$ to 525 $\mathrm{nm}$ ). Combining resonance Raman spectroscopy with density functional theory computations, we show a significant backbone planarization in the ordered phase. Moreover, the ratio of ordered phase to random-coil phase in PBS solution, as well as the extent of intermolecular interactions in the ordered phase, can be tuned by varying the temperature. Femtosecond transient absorption spectroscopy reveals that the excited-state behaviour of the polyelectrolyte is strongly affected by the degree of ordering. While triplet state formation is favoured in the random-coil chains, the ordered chains show a weak yield of polarons, related to interchain interactions. The investigated polyelectrolyte has been previously used as a biological DNA sensor, based on optical transduction when the conformation of the polyelectrolyte changes during assembly with the biomolecule. Therefore, our results, by correlating the photophysical properties of the polyelectrolyte to backbone and intermolecular conformation in a biologically relevant buffer, provide a significant step forward in understanding the mechanism of the biological sensing.
\end{abstract}




\section{INTRODUCTION}

Conjugated polymers, and in particular polythiophene derivatives, have emerged as important materials for organic electronics, including applications in photovoltaics, light-emitting diodes and field effect transistors. ${ }^{1-7}$ At the basis of this success, their favourable optoelectronic properties are of high scientific interest. P3HT (poly(3-hexylthiophene-2,5-diyl)), a state-of-the art solar cell material, has been extensively investigated in organic solvents and in solid-state thin films. It was shown that the absorption and emission spectra, as well as the excited-state properties, are strongly determined by the intrachain polymer conformation and by interchain aggregation. ${ }^{7-22}$ Conjugated polymers can be synthesised with ionic side-chains, yielding conjugated polyelectrolytes (CPEs), which combine the optoelectronic properties of $\pi$ conjugation in the backbone with solubility in highly polar solvents and the electrostatic behaviour of polyelectrolytes. ${ }^{23}$ CPEs provide the unique opportunity to manipulate the conformation of conjugated polymers in water and to correlate this to their photophysical behaviour. Besides the effects of solvent and temperature that have been thoroughly studied in non-ionic conjugated polymers, ${ }^{7-9,11,19,24}$ additional questions related to the variability in their optical properties with the nature and sign of the charged side chains, the solution $\mathrm{pH}$ and ionic strength in the aqueous medium can be addressed in CPEs.

Understanding the photophysical processes operative in CPEs associated with a certain backbone conformation is not only of high fundamental significance, but is also important both for optoelectronic as well as biological applications. Indeed, beyond their use in electronic devices, ${ }^{23,} 25$ the water-solubility and bio-compatibility of CPEs has been exploited in biological applications, for bioelectrochemistry, ${ }^{26}$ biosensing, ${ }^{27-29}$ cell imaging, ${ }^{30,31}$ drug delivery, ${ }^{32}$ as well as for anticancer and antimicrobial activity. ${ }^{33-35}$ The photophysics of CPEs play a key role in the action mechanism of those applications. In optoelectronic devices, CPEs can be used as thin injection layers between the electrodes and highly emissive neutral conjugated polymers ${ }^{36}$ or in hybrid devices with perovskites, ${ }^{37}$ therefore investigation of charge transfer states and their lifetimes is central to the operation of such devices. The detection of the triplet excited state of CPEs is crucial for the generation of singlet oxygen and subsequent light-activated antibacterial activity, ${ }^{38}$ while polaron formation and extension of their lifetime is an important target for cationic CPEs employed in artificial photosynthesis. ${ }^{39} \mathrm{CPE}$-based biosensing, which promises 
the development of simple biological assays, ${ }^{23,}{ }^{27-29}$ relies on strong changes in the optical properties of the CPE following selective assembly with the biomolecule of interest via electrostatic, $\pi$-stacking and H-bonding interactions. For instance, the cationic polythiophene derivative 1 (inset of Figure 1), to which we will refer to as CPT, forms well-defined complexes with anionic single DNA strands. ${ }^{40-44}$ Upon addition of the complementary DNA strand, hybridization is selectively transduced via a colour change, induced by conformational modifications in the CPT backbone.

Interestingly, assembly with DNA affects the photophysics of CPT in a sequence-dependent way, ${ }^{40}$ but a precise understanding of the DNA-induced conformational changes that lead to the observed optical effects is currently missing. Working towards this aim, we present here a spectroscopic study of CPT in the aqueous solution of interest, i.e. in phosphate buffered saline (PBS), a buffer commonly used when working with DNA and other biomolecules. In order to correlate the photophysical properties of CPT to chain conformation and aggregation, we have manipulated the structural aspects using salt and temperature effects. Even though the temperature range we use in our study extends outside the one suitable for biological applications, we consider temperature here as a stimulus that changes the polymer conformation to simulate the situation upon binding with different DNA sequences, focusing on understanding the photophysics of the polymer alone before delving into a more complicated system. Temperature-controlled order-disorder transitions have been previously studied in solutions of non-ionic polymers such as polythiophenes, ${ }^{9}, 45$ and analogues of PPV (poly(pphenylene vinylene)), ${ }^{46}, 47$ confirming that the intra- and interchain electronic coupling resulting from the molecular conformation and aggregation can be tuned with temperature. ${ }^{46}$ Some reports of thermochromic behavior have appeared about CPEs, ${ }^{48,}{ }^{49}$ however, those are limited to the stationary optical properties. Using stationary absorption, resonance Raman (RR) and femtosecond transient absorption (TA) spectroscopy, together with density functional theory (DFT) computations, we show that CPT adopts two types of conformations in PBS solution (random-coiled and ordered), the ratio of which can be controlled by temperature. We find that the conformation of CPT has a significant impact on the excited-state behaviour of the polyelectrolyte. Indeed, the dis-ordered phase of CPT leads to triplet state formation, while the ordered phase shows a weak yield of polarons following photo-excitation. 


\section{EXPERIMENTAL METHODS}

Materials. Cationic poly(1H-imidazolium,1-methyl-3-[2-[(4-methyl-3-thienyl)oxy]ethyl]-, chloride), abbreviated here as CPT (cationic polythiophene), was synthesised as previously described. ${ }^{43,}{ }^{44}$ It has a molecular weight $\left(\mathrm{M}_{\mathrm{w}}\right)$ of $22 \mathrm{kDa}\left(\mathrm{M}_{\mathrm{n}}=11 \mathrm{kDa}\right)$, with a polydispersity index (PDI) of 2.0. Each monomer unit of $262.8 \mathrm{~g} / \mathrm{mol}$ contains one positive charge on the ionic side-chain, compensated by a $\mathrm{Cl}^{-}$counter ion. A stock solution of CPT (monomeric concentration of $2 \cdot 10^{-3} \mathrm{M}$ ) was prepared in water (purified through a Synergy water purification system) and stored in the freezer. Phosphate buffered saline (PBS) was purchased from Thermo Fisher Scientific (catalogue number: 10010-031, pH 7.4, $\mathrm{KH}_{2} \mathrm{PO}_{4} 1.06$ mM, $\mathrm{Na}_{2} \mathrm{HPO}_{4} 2.97 \mathrm{mM}$, $\mathrm{NaCl} 155 \mathrm{mM}$ ) and was used to dilute the CPT stock solution to a monomeric concentration of $7.3 \cdot 10^{-5} \mathrm{M}$ for stationary absorption measurements and of $1.5 \cdot 10^{-4} \mathrm{M}$ for $\mathrm{TA}$ and $\mathrm{RR}$ experiments. The different concentrations had a negligible effect on the shape of the absorption spectrum (Figure S1 of the Supporting Information, S.I.).

Temperature control. Temperature control during absorption and TA measurements was achieved using a stand-alone Peltier-based temperature-controlled cuvette holder (Flash300/E, Quantum Northwest). The cuvette was placed inside the holder, set to the required temperature and the temperature was allowed to stabilize for 5-10 min. A small magnetic stir bar was placed in the cuvette and the stirring speed could be controlled with the cuvette holder. Below $10^{\circ} \mathrm{C}$, nitrogen was flushed inside the cuvette holder in order to avoid condensation on the cuvette.

Stationary absorption spectroscopy. Absorption spectra were recorded with a UV/VIS/NIR Lambda 900 spectrometer (Perkin-Elmer). A quartz cuvette with an optical path length of $10 \mathrm{~mm}$ (Starna Cells Inc.) was placed inside the temperature-controlled holder.

Transient Absorption Spectroscopy. Transient absorption (TA) spectra were recorded using femtosecond pulsed laser pump-probe spectroscopy. The solutions were placed in a quartz cuvette with an optical path length of $2 \mathrm{~mm}$ (Starna Cells Inc.) placed inside the temperaturecontrolled holder and held by a piece of aluminium. Pump excitation at $400 \mathrm{~nm}$ (200 fs resolution) was achieved by frequency doubling the fundamental $800 \mathrm{~nm}$ laser output (from a Ti:sapphire laser system with regenerative amplification providing $35 \mathrm{fs}$ pulses at a repetition rate of $1 \mathrm{kHz}$, Astrella, Coherent). As an alternative, an excitation beam at $580 \mathrm{~nm}(<100 \mathrm{fs}$ 
resolution) was generated with a commercial optical parametric amplifier (OPerA Solo, Coherent). The pump diameter was about $1 \mathrm{~mm}$ and the pump intensity was varied between 150 and $1500 \mathrm{~nJ}$ to keep the number of absorbed photons constant. The probe beam consisted of a white light continuum ( 450-1200 nm) generated by passing a portion of the $800 \mathrm{~nm}$ amplified Ti:sapphire output through a $5 \mathrm{~mm}$ thick sapphire window. Either $720 \mathrm{~nm}$ low pass filters or 850 $\mathrm{nm}$ long pass filters were used to remove the remaining fundamental intensity from the white light, when the visible and the near-infrared (nIR) parts of the spectrum were recorded separately. The probe intensity was negligible compared to the pump intensity and the spot size was much smaller (probe energy of $<5 \mathrm{~nJ}$, probe diameter of about $160 \mu \mathrm{m}$ ). The probe pulses were time delayed with respect to the pump pulses by means of a computer-controlled translation stage. The probe beam was split before the sample into a signal beam (transmitted through the sample and overlapped with the pump beam) and a reference beam. The signal and reference beams were detected separately using a pair of spectrographs (home-built prism spectrometers) equipped with 512 x 58 pixels back-thinned CCDs (Hamamatsu S07030-0906) and assembled by Entwicklungsbüro Stresing, Berlin. The pump beam was chopped at half the amplifier frequency to improve the sensitivity of the set-up. The transmitted intensity of the signal beam was recorded shot-by-shot and it was corrected for laser intensity fluctuations using the reference beam. The single shot TA spectra were averaged 3000 times at each time delay and the entire range of measured time delays (between -4 ps and $1 \mathrm{~ns}$ ) was scanned 5 times, without any noticeable signs of degradation. Wavelength calibration was accomplished with a series of $10 \mathrm{~nm}$ bandpass filters. To avoid polarization effects, the relative polarization of the probe and pump pulses was set at the magic angle. All spectra were corrected for the chirp of the white-light probe. MATLAB and IgorPro software were used for data analysis.

Resonance Raman Spectroscopy. The samples were excited at 405 and $473 \mathrm{~nm}$ with CW diode lasers (MonopowerTM 405-50-MM, Alphalas, and Ultralasers, $473 \mathrm{~nm}, 50 \mathrm{~mW}$ OEM DPSS Laser). The excitation light was focused into a spinning cell consisting of an EPR fused silica tube (diameter: $4 \mathrm{~mm}$ ) attached to a rheostat-controlled motor for choice of rotation speed. Use of the spinning cell prolonged the lifetime of the samples. Very modest excitation powers $(2.3$ $\mathrm{mW}$ ) were employed to avoid decomposition of the sample. For temperature-dependent measurements, the tube was placed in a thermal mantle that was thermostated by a water bath at the desired temperature. The Raman scattered light was collected in a backscattering geometry 
and delivered to a $0.75 \mathrm{~m}$ focal-length Czerny-Turner spectrograph (SpectraPro, SP2760i, Princeton Instruments), equipped with a 1200-grooves/mm UV-enhanced holographic grating. The slit width was set to $100 \mu \mathrm{m}$ providing for $5 \mathrm{~cm}^{-1}$ spectral resolution at the wavelengths used in this work. The scattered light was detected by a $\mathrm{LN}_{2}$-cooled $2048 \times 512$ pixel, back-illuminated UV-enhanced CCD detector (Spec10:2KBUV/LN, Princeton Instruments). Each spectrum presented here is the accumulation of $12 \times 5$ min spectra. Frequency calibration of the spectra was accomplished with the use of cyclohexane. MATLAB and ORIGIN software were used for spectral treatment and analysis.

Computational details. The effects of the polymer backbone planarity on the Raman spectra and absorption energies were investigated using a set of geometries of substituted quaterthiophenes optimized under constrain. The geometries were based on the scan of the wobble $\left[0,180^{\circ}\right]$ torsion mode of the three $\angle \mathrm{SCCS}$ dihedral angles $(\theta)$. Chlorine anions were added at $3 \AA$ from the geometric center of each charged imidazole ring, located in the side chain. Geometries were optimized in gas phase at the B3LYP-D3(BJ)/6-31G(d) level, keeping the three $\angle$ SCCS angle frozen at the same $\theta$ value. Raman spectra were computed at the B3LYP/6-31G(d) level ${ }^{50}$; peaks were broadened with a full width at half maximum of $30 \mathrm{~cm}^{-1}$, and the spectra were scaled according to $I_{v_{C_{\alpha}}=C_{\beta}}=1.0$. Absorption energies were computed at the CAMB3LYP/6-311G(d) level ${ }^{51}$. All computations were performed in the Gaussian09 code. ${ }^{52}$

\section{RESULTS}

\section{Temperature-Dependent Absorption Spectra}

The stationary absorption spectra at room temperature $\left(25^{\circ} \mathrm{C}\right)$ for CPT in pure water and in PBS buffer solution are shown in Figure 1a. In water, CPT displays an unstructured broad absorption band peaking at $402 \mathrm{~nm}(3.08 \mathrm{eV})$, in agreement with a previous report. ${ }^{53}$ A similar broad absorption (with maximum in the 440-450 nm region) is typically observed at room temperature for dilute P3HT solutions in good organic solvents such as chloroform, tetrahydrofuran or chlorobenzene. 7, 9, 11, 14, 19, 21 In analogy to the P3HT data, we ascribe the absorption of aqueous CPT solution to an intrachain $\pi-\pi^{*}$ transition in polymer chains having disordered random-coil conformations. The blue-shift of the CPT absorption in water compared to P3HT in solvents 
such as chlorobenzene is mostly due to solvatochromic effects, which are dominated by dispersion interactions between the polyelectrolyte backbone and the solvent (Figure S2 of the S.I. shows the dependence of the CPT absorption on the solvent refractive index). ${ }^{53}$ When CPT is dissolved in the PBS buffer solution, the broad absorption band around $402 \mathrm{~nm}$ is much less pronounced; instead the spectrum is dominated by a red-shifted absorption band with clear vibronic structure and peaks at $565 \mathrm{~nm}(2.19 \mathrm{eV}, 0-0$ transition), $525 \mathrm{~nm}$ (2.36 eV, 0-1 transition) and $\sim 495 \mathrm{~nm}(2.50 \mathrm{eV}$ ) (Figure 1a). The maximum at room temperature is at the 0-1 peak, and the spacing between the 0-0 and the 0-1 transitions corresponds to $\sim 168 \mathrm{meV}\left(1355 \mathrm{~cm}^{-1}\right)$, which can be attributed to the symmetric $\mathrm{C}=\mathrm{C}$ stretching and $\mathrm{C}-\mathrm{C}$ intra-ring vibrational modes of the thiophene units (see discussion of the Raman modes below). Again by comparison to P3HT, where a similar red-shifted structured absorption is present in poor solvents or solid-state thin films (with the 0-0 transition typically above $600 \mathrm{~nm}$ ), ${ }^{7}, 10,14,16,19,21$ the structured absorption band for CPT in PBS solution is assigned to more ordered polymer chains, having more planar and extended backbone conformations, and possibly showing interchain interactions in aggregates, as further elaborated in the Discussion. Inspection of the absorption spectra of CPT in solutions of the separate salts present in PBS at the relevant concentration shows that mainly the $\mathrm{Cl}^{-}$anions, which are present at a high concentration of $155 \mathrm{mM}$, are at the origin of the important spectral changes in the presence of the buffer (Figure 1a). As shown in Figure S3 of the S.I., a much weaker ordering effect was achieved at low $\mathrm{NaCl}$ concentration $(1.06 \mathrm{mM})$ or at high $\mathrm{Na}_{2} \mathrm{HPO}_{4}$ concentration $(155 \mathrm{mM})$, showing that it is specifically the high concentration of $\mathrm{Cl}^{-}$(and not of $\mathrm{Na}^{+}$or total ionic strength) that induces the observed spectral changes.

To gain more insight to the conformation-induced optical properties of CPT in PBS buffer solution, we carried out temperature-dependent absorption measurements (from $1^{\circ} \mathrm{C}$ to $90^{\circ} \mathrm{C}$ ). The spectra are shown in Figure $1 \mathrm{~b}$ (for clarity, different temperature ranges are depicted separately in Figure S4). Data was recorded upon cooling the solution, but any hysteresis with respect to the prior heating is negligible (Figure 1c, Figure S5). At the higher investigated temperatures, i.e. between $90^{\circ} \mathrm{C}$ and $70^{\circ} \mathrm{C}$, only the broad signature of the disordered randomcoil polymer chains is seen. In this range, there is a small red-shift (from $390 \mathrm{~nm}$ to $392 \mathrm{~nm}$ ) and increase in intensity $(<1 \%)$ of the absorption band with decreasing temperature. Based on previous work, we assign this mainly to a decrease of torsional disorder of the polymer chains in the random-coil phase (leading to an increase in conjugation length), ${ }^{9}$ and to temperature- 
induced changes in the solvent refractive index..$^{11}$ Below $70^{\circ} \mathrm{C}$, the red-shift of the structureless absorption band continues to $\sim 430 \mathrm{~nm}$ at $20^{\circ} \mathrm{C}$ (yielding a total shift of $296 \mathrm{meV}$ between $90^{\circ} \mathrm{C}$ and $20^{\circ} \mathrm{C}$, Figure 1c). At the same time, the intensity of this absorption band (representative of the random-coil conformations) decreases and the intensity of the structured absorption band above $450 \mathrm{~nm}$ (due to the more ordered polymer chains) concomitantly increases. A clear isosbestic point between the two spectral signatures is only visible below $20^{\circ} \mathrm{C}$, because it is masked by the spectral red-shift of the high-energy (random-coil) band that takes place down to $20^{\circ} \mathrm{C}$, in parallel to the conversion between the random-coil and more ordered conformations, and by the high polydisperisity of the polymer (see Discussion). ${ }^{9}$ The temperature evolution of the absorption intensity at $395 \mathrm{~nm}$ (random-coil) and $571 \mathrm{~nm}$ (ordered chains) in Figure 1c reveals that the conversion occurs over a broad temperature range (between $55^{\circ} \mathrm{C}$ and $5^{\circ} \mathrm{C}$ ). At the lowest measurable temperature of $1{ }^{\circ} \mathrm{C}$ the $\mathrm{CPT}$ chains are essentially in the more ordered conformations, and even the absorption around $400 \mathrm{~nm}$ has a predominant contribution of more ordered species (as further shown by the TA data below). Interestingly, we observe changes in the shape of the low-energy absorption band corresponding to the ordered CPT chains, when the temperature is decreased. At $50^{\circ} \mathrm{C}$ the $0-0$ vibronic peak is suppressed, but as the temperature is further reduced, there is a weak red-shift of the $0-0$ band (by $\sim 50 \mathrm{meV}$ between $45^{\circ} \mathrm{C}$ and $1{ }^{\circ} \mathrm{C}$ ) and an important increase of the relative intensity of the $0-0$ peak with respect to the $0-1$ peak (Figure $1 \mathrm{~b}, \mathrm{c}$ ). At $1{ }^{\circ} \mathrm{C}$, the $0-0$ to $0-1$ peak ratio is close to 1 . The observed spectral shifts for both the disorder and ordered species and the changes in the $0-0$ to $0-1$ peak ratio of the ordered phase hamper deconvolution of the spectra into the contributions of random-coil and ordered polymer chains, as has been reported in previous studies. ${ }^{9,} 16$ In pure water (Figure S6), the redshift and increase of intensity (by 12\%) of the broad $\sim 400 \mathrm{~nm}$ absorption band due to an increase of the conjugation length in the random-coil polymer chains with decreasing temperature is very pronounced. However, the signature of the low-energy structured absorption band only appears very weakly below $15^{\circ} \mathrm{C}$, indicating that the conversion from the random-coil conformation to more ordered species does not efficiently take place in the accessible temperature range (above freezing), as chain ordering is not favoured without PBS. 

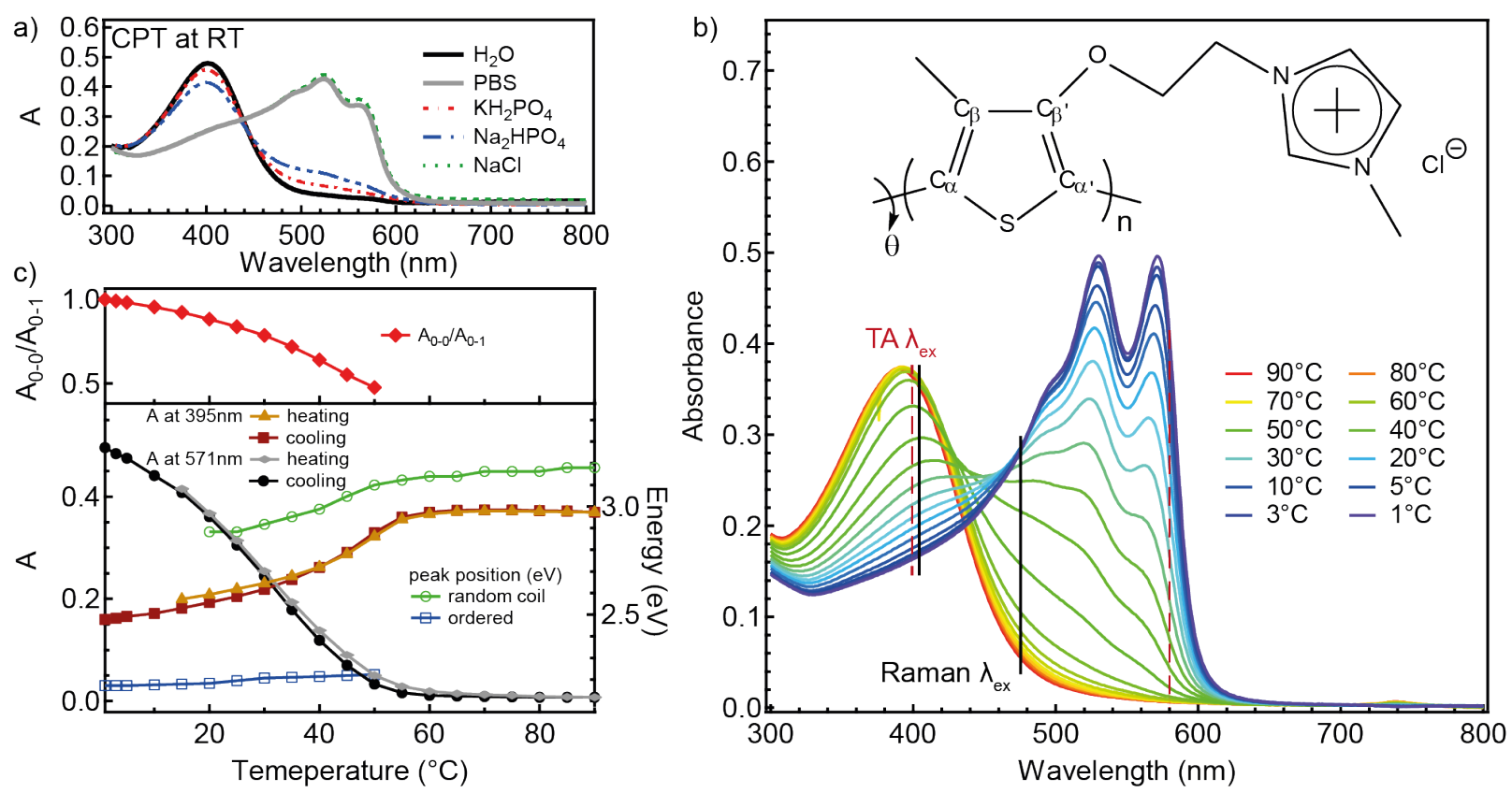

Figure 1. (a) Absorption spectra of CPT $\left(7.3 \cdot 10^{-5} \mathrm{M}\right.$, monomeric basis) in pure water, in PBS buffer and in different salt solutions at the relevant concentration of $\mathrm{PBS}\left(\mathrm{KH}_{2} \mathrm{PO}_{4} 1.06 \mathrm{mM}\right.$, $\mathrm{Na}_{2} \mathrm{HPO}_{4} 2.97 \mathrm{mM}$ and $\mathrm{NaCl} 155 \mathrm{mM}$ ) at room temperature. (b) Absorption spectra of CPT in PBS at different temperatures between $1{ }^{\circ} \mathrm{C}$ and $90^{\circ} \mathrm{C}$. Excitation wavelengths for TA (red dashed lines) and RR (black solid lines) measurements are also shown. Inset: Chemical structure of CPT. (c) Spectral characteristics of CPT in PBS as a function of temperature: $A_{0-0} / A_{0-1}$ ratio (top), absorbance upon heating and cooling extracted at 395 and $571 \mathrm{~nm}$ (bottom, left axis) and peak position of the broad random-coil red-shifted absorption and of the $0-0$ peak of the structured blue-shifted absorption of the ordered chains (bottom, right axis).

\section{Resonance Raman Studies}

We have explored the conformational changes of CPT in PBS buffer solution as a function of temperature using resonance Raman spectroscopy. We have excited the polymer at two different wavelengths (405 and $473 \mathrm{~nm}$, see Figure 1b), in order to probe the disordered coiled and more ordered CPT conformations, respectively. We begin with the room temperature RR spectra of $\mathrm{CPT}$ in PBS in the fingerprint region excited at $405 \mathrm{~nm}$ (top of Figure 2a, Figure S7). Two intense bands are observed at 1498 and $1405 \mathrm{~cm}^{-1}$, while weaker bands are observed as shoulders at 1365,1454 , and $1534 \mathrm{~cm}^{-1}$, and in the lower wavenumber region at $\sim 800,885$ and $965 \mathrm{~cm}^{-1}$. We have carried out DFT computations in a gas phase model of a CPT tetramer (see computational details), in order to assign the Raman bands, as most of the Raman literature on 
oligothiophenes and polythiophenes has focused on non-substituted thiophenes or alkyl substituted thiophenes, such as P3HT, but not on polythiophenes with cationic side-chains. In agreement with this previous literature, ${ }^{54-60}$ the computed Raman spectra, shown at the bottom of Figure $2 \mathrm{a}$ for different conformers, are dominated by two main vibrational bands $\left(v_{2}\right.$ and $\left.v_{6}\right)$, which originate from the $C_{\alpha}=C_{\beta}$ thiophene ring bond stretch and the $C_{\beta}-C_{\beta}$, ring stretch mixed with the $\mathrm{C}_{\alpha}-\mathrm{C}_{\alpha}$, inter-thiophene stretch, respectively, with participation of $\mathrm{C}-\mathrm{H}$ bending in the side chains (see Table 1 and Figure S8.1-S8.6 for detailed spectra). Small differences in the computed features and the band frequencies reported in previous work on P3HT emerge from steric hindrance and the strong coulombic interactions between the side chains, which induce distortions of the thiophene backbone (see optimized structures in Figure S8.7). Previous reports show that alkoxy substitution at the 3 position of the thiophene causes splitting of the $\mathrm{C}_{\alpha}=\mathrm{C}_{\beta}$ symmetric stretch band to one assigned to the $\mathrm{C}_{\alpha}=\mathrm{C}_{\beta}(\mathrm{H})$ and one to the $\mathrm{C}_{\alpha}=\mathrm{C}_{\beta}(\mathrm{O})$ stretch, with the latter at lower frequencies than the former, which remains at similar frequencies as in alkylsubstituted polythiophenes. ${ }^{56,57}$ This vibration is visible in the computed spectra and is associated with the $v_{3}$ band (see Figure S8.1-S8.6 for more resolved and detailed spectra). Based on the computations, the 1498 and $1405 \mathrm{~cm}^{-1}$ bands in the experimental spectra are, therefore, assigned to the thiophene $\mathrm{C}_{\alpha}=\mathrm{C}_{\beta}(\mathrm{H})$ symmetric stretch and the intra-ring $\mathrm{C}_{\beta}-\mathrm{C}_{\beta}$, stretch, respectively. The shoulder observed at $1454 \mathrm{~cm}^{-1}$ is assigned to the $\mathrm{C}_{\alpha}=\mathrm{C}_{\beta}(\mathrm{O})$ stretch and the weak band at $1534 \mathrm{~cm}^{-1}$ corresponds to the $\mathrm{C}_{\alpha}=\mathrm{C}_{\beta}$ asymmetric stretch. The low frequency modes at 885 and $965 \mathrm{~cm}^{-1}$ are assigned to ring $\mathrm{C}-\mathrm{S}$ stretching and ring- $\mathrm{CH}_{3}$ rocking, respectively, in agreement with previous work on a methyl-substituted polythiophene. ${ }^{61,}{ }^{2}$ Similar RR spectra have been reported for another polythiophene polyelectrolyte with an alkoxy cationic side chain, the cation being a tertiary amine. ${ }^{63}$

Upon excitation at $473 \mathrm{~nm}$, the RR spectrum of CPT in PBS significantly changes, with the $\mathrm{C}_{\alpha}=\mathrm{C}_{\beta}$ stretch band downshifted to $1487 \mathrm{~cm}^{-1}$, and the relative intensity between the $\mathrm{C}_{\alpha}=\mathrm{C}_{\beta}$ and the $\mathrm{C}_{\beta}-\mathrm{C}_{\beta}$, stretch modes reversed with the latter being more intense at this wavelength (middle of Figure 2a, at room temperature). A significant narrowing of the vibrational bands is observed here, with the $v_{3}$ band at $1448 \mathrm{~cm}^{-1}$, assigned to the $\mathrm{C}_{\alpha}=\mathrm{C}_{\beta}(\mathrm{O})$ stretch, becoming more pronounced. The change in relative intensity of the $C_{\beta}-C_{\beta}$, to the $C_{\alpha}=C_{\beta}$ mode has been reported previously as an indicator of the planarity of the polymer backbone. ${ }^{60,64}$ The effect of the dihedral angle variation on the Raman spectrum is evaluated computationally here for the CPT 
tetramer through a scan of the $\angle \mathrm{SCCS}$ dihedral angle $(\theta)$. As for pure polythiophene, the frequency of the CPT $\mathrm{C}_{\alpha}=\mathrm{C}_{\beta}$ stretching band strongly varies with the planarity of the thiophene cores (see Figure $2 \mathrm{a}, \mathrm{d}$ ): the lower the planarity $\left(\theta \rightarrow 90^{\circ}\right)$, the higher the $v_{2}$ frequency. This effect is caused by the lower inter-ring electronic conjugation when adjacent thiophene rings become non-planar, thus reinforcing the $\mathrm{C}_{\alpha}=\mathrm{C}_{\beta}$ bond and increasing its associated frequency $\left(v_{2}\right)$. Hence, a broad experimental band is expected in disordered structures, whereas sharper $v_{2}$ bands should be present at lower frequencies in case of highly planar structures. Besides, the $\frac{I_{v_{c}=c}}{I_{v_{c}-c}}$ Raman intensity ratio decreases at high planarity $\left(\theta \rightarrow 0^{\circ}\right.$ or $180^{\circ}$, see Figure $2 \mathrm{~d}$ and Figure S9 for the whole range of $\theta$ from $0^{\circ}$ to $180^{\circ}$ ), in agreement with computations for P3HT by Tsoi et al. ${ }^{60}$ In addition, the shoulder observed experimentally at $1448 \mathrm{~cm}^{-1}$ ( $v_{3}$ vibration), which is strongly enhanced at $\lambda_{\mathrm{ex}}=473 \mathrm{~nm}$, is increasing for the more planar computed systems (see detailed spectra in Figures S8.1-S8.6). In light of these computations, we conclude that excitation of CPT in PBS at $473 \mathrm{~nm}$ probes a subset of the polymer population consisting of more planar conformers, as evidenced from the increased relative intensity of the $C_{\beta}-C_{\beta}$, to the $C_{\alpha}=C_{\beta}$ band, the narrower bandwidths and the $\sim 10 \mathrm{~cm}^{-1}$ frequency downshift of the $\mathrm{C}_{\alpha}=\mathrm{C}_{\beta}$ band. Based on the computations in Figure 2d and Figure S9, we estimate an average diedral angle change of 20-30 compared to $405 \mathrm{~nm}$ excitation, making the backbone almost completely planar.

Figure $2 \mathrm{~b}$ presents the temperature-dependent RR spectra of CPT in PBS in the range $8-55^{\circ} \mathrm{C}$ with excitation at $405 \mathrm{~nm}$. The spectra were normalized with respect to the $1498 \mathrm{~cm}^{-1}$ band for comparison. With increasing temperature, a decrease is observed in the intensity of the $1405 \mathrm{~cm}^{-1}$ mode $\left(v_{6}\right)$ relative to the $\mathrm{C}_{\alpha}=\mathrm{C}_{\beta}$ symmetric stretch $\left(v_{2}\right)$, indicative of an increase in torsional disorder. A slight increase in the frequency of the $\mathrm{C}_{\alpha}=\mathrm{C}_{\beta}$ symmetric stretch and decrease in the magnitude of $v_{3}\left(\mathrm{C}_{\alpha}=\mathrm{C}_{\beta}(\mathrm{O})\right.$ stretch $)$ are seen as well, which agrees with the increase in torsional disorder. This conformational change is relatively small, with a change in the average planarity between thiophene units of less than $10^{\circ}$ according to our computations (Figure $2 \mathrm{~d}$ and Figure S9). We also computed the vertical transition energies in the model tetramer as a function of $\theta$, confirming the red-shift of the stationary absorption spectrum with increasing planarity, which we observed experimentally for both the random-coil and ordered steady-state absoprtion bands (Figure 1a). An energy shift of $\sim 0.3 \mathrm{eV}$ is observed on the UV peak maxima (random-coil phase) when passing from 8 to $55^{\circ} \mathrm{C}$, which corresponds to a change of $\sim 8^{\circ}$ in polymer planarity, when 
taking the linear regime of the computational plot (Figure $2 \mathrm{~d}$ and Figure S9). The stationary absorption points thus to a similar conformational change as the RR results. Excitation of CPT at $473 \mathrm{~nm}$ becomes difficult with an increase in temperature due to the increased fluorescence background caused by emission of the random-coil chains. We can see, however, that the spectrum remains almost unaltered up to $40^{\circ} \mathrm{C}$, with only a slight decrease in the $\mathrm{C}_{\beta}-\mathrm{C}_{\beta}$, band intensity (Figure 2b). This corroborates the conclusion that at this excitation wavelength, a subset of particularly planar and extended polymer chains is excited independently of the temperature.

Table 1: Band assignment of the experimental RR peaks of CPT recorded at room temperature with different excitation wavelengths $(405 \mathrm{~nm}, 473 \mathrm{~nm})$, according to the computations on model tetrameric conformers obtained at the B3LYP/6-31G* level, on geometries optimized at the B3LYP-D3(BJ)/6-31G* level, keeping the three SCCS dihedral angles $(\theta)$ frozen at $0^{\circ}, 90^{\circ}$ and $180^{\circ}$, respectively. Computed frequencies refer to individual vibrational modes, and are corrected using the empirical scaling factor for B3LYP (0.97). ${ }^{65}$

\begin{tabular}{ccccccc}
\hline & & \multicolumn{5}{c}{ Frequency $\left(\mathrm{cm}^{-1}\right)$} \\
Label & Assignment & Computed & Computed & Computed & Expt. & Expt. \\
& & $\theta=0^{\circ}$ & $\theta=90^{\circ}$ & $\theta=180^{\circ}$ & $405 \mathrm{~nm}$ & $473 \mathrm{~nm}$ \\
\hline$v_{1}$ & $\mathrm{C}_{\alpha}=\mathrm{C}_{\beta}$ ring stretching (anti) & 1545,1531 & 1577 & 1566,1543 & 1534 & 1517 \\
$v_{2}$ & $\mathrm{C}_{\alpha}=\mathrm{C}_{\beta}(\mathrm{H})$ ring stretching & 1460 & 1523 & 1485 & 1498 & 1487 \\
$v_{3}$ & $\mathrm{C}_{\alpha}=\mathrm{C}_{\beta}(\mathrm{O})$ ring stretching & 1454 & 1507 & 1461 & 1454 & 1448 \\
$v_{4}$ & $\mathrm{C}_{\beta}-\mathrm{C}_{\beta^{\prime}}$ ring stretching & 1419 & 1420 & 1443 & --- & --- \\
$v_{5}$ & $\mathrm{C}_{\beta}-\mathrm{C}_{\beta^{\prime}}$ ring stretching & 1403 & 1407 & 1413 & --- & --- \\
$v_{6}$ & Mixed $\mathrm{C}_{\beta}-\mathrm{C}_{\beta^{\prime}}$ and $\mathrm{C}_{\alpha}-\mathrm{C}_{\alpha^{\prime}}$ & 1352 & 1384 & 1383 & 1405 & 1401 \\
stretching & & & & & & \\
\hline
\end{tabular}



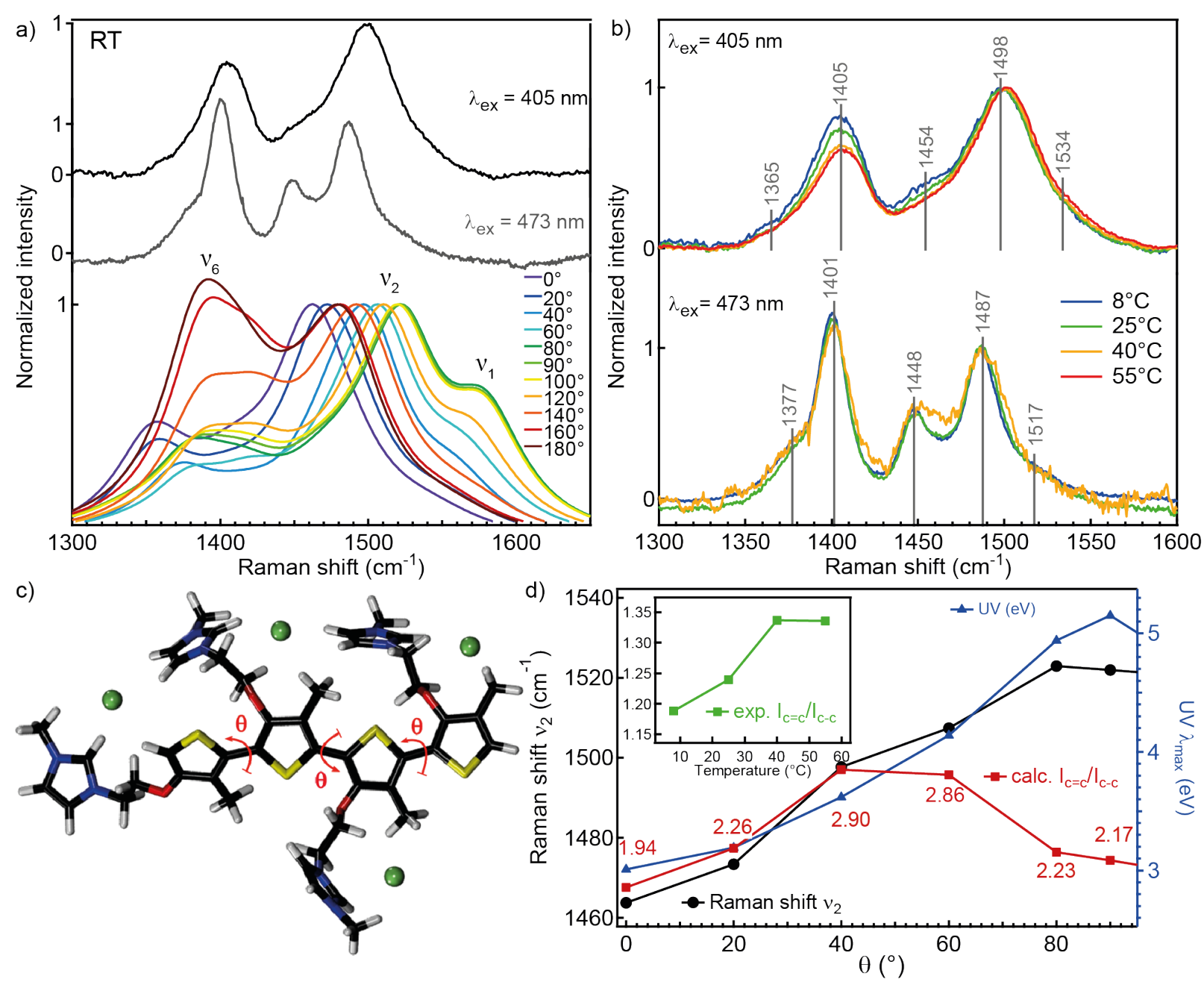

Figure 2. (a) Top: Experimental Resonance Raman spectra of CPT in PBS solution $\left(1.5 \cdot 10^{-4} \mathrm{M}\right.$, monomeric basis) at room temperature excited at $405 \mathrm{~nm}$ and $473 \mathrm{~nm}$. Bottom: Raman spectra of the "wobble" structures of the CPT tetramer computed at the B3LYP /6-31G* level; energies corrected using an empirical scaling factor for B3LYP (0.97). ${ }^{65}$ Geometries were optimized at the B3LYP-D3(BJ)/6-31G* level, keeping the three SCCS dihedral angles $(\theta)$ frozen at the color coded value. (b) RR spectra of CPT in PBS solution at different temperatures at both excitation wavelengths. (c) Geometry optimized at $\theta=160^{\circ}$ with the "wobble" torsion mode indicated. (d) Dihedral angle dependence of the computed $v_{2}$ band maximum, of the computed $\frac{I_{v}=c}{I_{v}-c}$ Raman intensity ratio and of the vertical excitation energies (computed at the CAM-B3LYP/6$311 \mathrm{G}^{*} \mathrm{level}$ ). The inset shows the experimental $\frac{I_{v}=c}{I_{v_{c}-c}}$ ratio as a function of temperature. (for the full range from 0 to $180^{\circ}$, see Figure S9) . 


\section{Transient Absorption Studies}

\section{0 nm Excitation}

A selection of TA spectra for CPT in PBS buffer solution at $5^{\circ} \mathrm{C}$ and $55^{\circ} \mathrm{C}$ at different time delays following excitation at $400 \mathrm{~nm}$ is shown in Figure 3a. At high temperature, the $400 \mathrm{~nm}$ excitation falls into the unstructured high-energy absorption band (Figure 1b) and therefore excites the random-coil conformations of CPT. As we will show below, at low temperature, the more ordered phase is contributing significantly to the TA spectra even with excitation at 400 $\mathrm{nm}$. Let us start by considering the data at $5^{\circ} \mathrm{C}$ (Figure 3a, top part). The structured negative band in the 450-600 $\mathrm{nm}$ region is attributed to the ground state bleaching (GSB) because of the similarity with the stationary absorption spectrum (black curve), with vibronic peaks at $571 \mathrm{~nm}$, $528 \mathrm{~nm}$ and a shoulder at $\sim 490 \mathrm{~nm}$. This structured GSB is present within the 200 fs time resolution of our experiment, providing a strong indication that the ordered conformations of CPT are populated at $400 \mathrm{~nm}$. Significant presence of any random-coil conformations remaining at $5^{\circ} \mathrm{C}$ would, on the other hand, lead to an unstructured, blue-shifted GSB. The $571 \mathrm{~nm}$ peak is not only due to the GSB, but also due to some overlap with stimulated emission (SE). Indeed, the ratio between the $0-0$ and $0-1$ vibronic peaks in the stationary absorption spectrum (0.98) is significantly lower than the corresponding ratio in the TA spectra (1.26 at $0.2 \mathrm{ps}$ ), showing that the SE enhances the negative intensity in the region of the 0-0 peak. Similarly, the small "negative" indent in the TA spectra at $\sim 650 \mathrm{~nm}$, overlapping with a broad positive band, is the 01 peak of the SE. The broad positive band reaching from $600 \mathrm{~nm}$ to $1250 \mathrm{~nm}$ and centred at $\sim 1050 \mathrm{~nm}$ is present immediately after excitation and corresponds to the excited state absorption (ESA) of the $\mathrm{S}_{1}$ singlet excited state. A similar broad feature has been observed for P3HT in solution and thin films with the maximum situated between 1000-1200 nm, depending on the degree of order and on the conjugation length (see Discussion section). ${ }^{15,} 18$ We note that the small oscillations around $850 \mathrm{~nm}$ are an artefact in the white light generation near the fundamental $800 \mathrm{~nm}$ laser output. At longer time delays, the ESA and SE signatures decrease and completely disappear, concomitantly with a partial decay of the GSB. The normalised dynamics of the ESA at $1150 \mathrm{~nm}$ is shown in the inset of the upper part of Figure 3a and decays with time constants of 2.1 ps, 37 ps and 450 ps. Such multiphasic excited-state decay, entangled with early relaxation processes, is typical for P3HT and other conjugated polymers. ${ }^{14,15,66}$ After 
about 500 ps, only weak signals of the GSB and of a new positive absorption band, which is relatively flat above $600 \mathrm{~nm}$ and slightly peaks at $\sim 955 \mathrm{~nm}$, remain in the TA spectra. This is also evident by the long-lived offset in the dynamics probed in the GSB $(530 \mathrm{~nm})$ and at $955 \mathrm{~nm}$, as shown in the upper inset of Figure 3a. Based on the similarity of the spectral shape of the long-lived absorption with the one reported for positively charged polythiophene chains in P3HT and P3HT:fullerene films, ${ }^{15,67-69}$ we assign the long-lived signature to polarons formed in CPT.
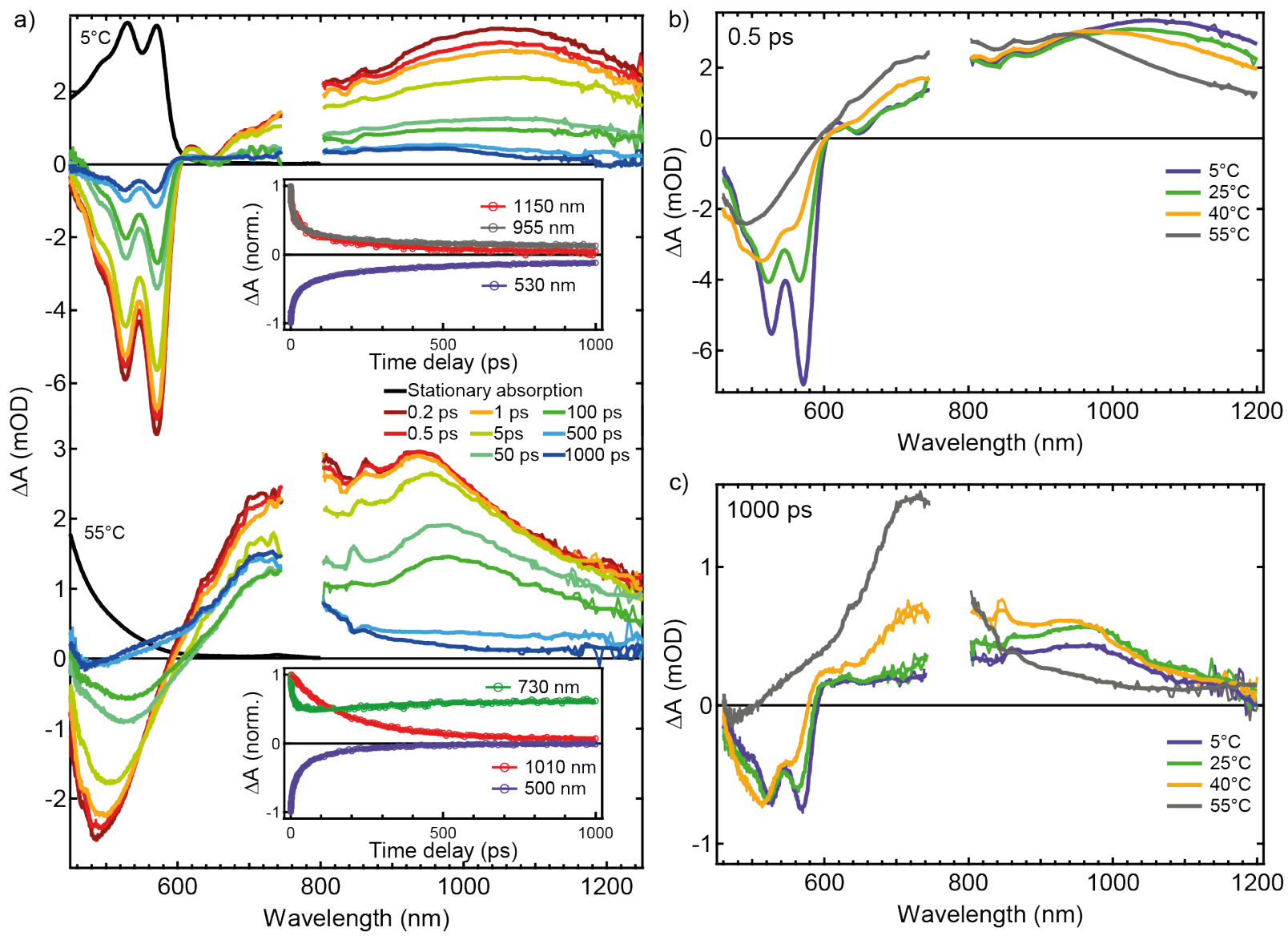

Figure 3. TA data for CPT in solution following excitation at $400 \mathrm{~nm}\left(1.5 \cdot 10^{-4} \mathrm{M}\right.$ on a monomeric unit basis in PBS). (a) TA spectra recorded at selected time delays for $\mathrm{CPT}$ at $5^{\circ} \mathrm{C}$ (top) and $55^{\circ} \mathrm{C}$ (bottom) with the corresponding stationary absorption (black curves). The two insets show the TA dynamics probed at selected wavelengths. (b) TA spectra at four temperatures $\left(5,25,40\right.$ and $\left.55^{\circ} \mathrm{C}\right)$ at $0.5 \mathrm{ps}$ after photoexcitation. (c) TA spectra at the four temperatures at $1000 \mathrm{ps}$ after photoexcitation. 
Let us now turn to the TA spectra for CPT in PBS recorded at $55^{\circ} \mathrm{C}$ with $400 \mathrm{~nm}$ excitation, where mainly the random-coil phase is excited (Figure 3a, bottom part). We note that the pump fluence was adjusted to maintain the number of absorbed photons constant at all temperatures. The TA spectra below $600 \mathrm{~nm}$ are dominated by a negative band that is red-shifting with time,

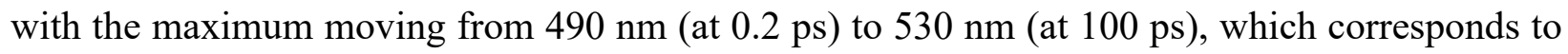
a difference in energy of $190 \mathrm{meV}$. This feature is ascribed to SE, since the stationary fluorescence spectrum of CPT in aqueous solution at $55^{\circ} \mathrm{C}$ is known to be centred at $530 \mathrm{~nm} .{ }^{44}$ The stationary absorption spectrum is however mainly outside the TA window (at around 400 $\mathrm{nm}$, Figure 1b), so that only the tail of the GSB weakly contributes to the negative TA feature. The broad positive band between $\sim 600 \mathrm{~nm}$ and $1250 \mathrm{~nm}$ is due to ESA from the singlet excited state. This band is also red-shifting with time, from $940 \mathrm{~nm}$ (at $0.2 \mathrm{ps)}$ to $980 \mathrm{~nm}$ (at $100 \mathrm{ps),}$ which corresponds to a difference in energy of $\sim 54 \mathrm{meV}$. A red-shift of the time-resolved fluorescence, SE and ESA on this time scale is typical for conjugated polymers and has been extensively studied for various polythiophene solutions and thin films. ${ }^{11,14,20,70}$ It is caused by excitation energy transfer from shorter to the longer polymer segments and by torsional relaxation. Indeed, CPT in the random-coil conformation consists of an inhomogeneous ensemble of conjugated segments of different lengths and energies, leading to a gradual downenergy migration of the excitation. At the same time, there is slow torsional planarization of the backbone towards a more conjugated, lower energy structure. The spectral relaxation in CPT at $55^{\circ} \mathrm{C}$ leads to fast dynamics with time constants of $0.9 \mathrm{ps}, 6.6 \mathrm{ps}$ and $44 \mathrm{ps}$ in the SE (probed at $500 \mathrm{~nm}$ ), entangled with slower decay of the feature within tens to hundreds of picoseconds, as shown in the bottom inset of Figure 3a. We note that the red-shift of the SE and ESA is not observed for CPT at $5^{\circ} \mathrm{C}$, which reflects the more homogeneous conjugation length and lower torsional disorder at low temperature. At longer time delays, the SE and ESA signatures entirely disappear for $\mathrm{CPT}$ at $55^{\circ} \mathrm{C}$ and a new slightly narrower band rises, extending from $\sim 500-1100$ $\mathrm{nm}$ with a pronounced peak at $730 \mathrm{~nm}$ (bottom of Figure 3a). Based on previous work on polythiophenes, it is attributed to the $\mathrm{T}_{1}$ triplet state of CPT formed from the singlet state by

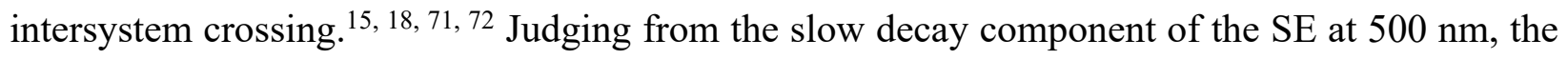
decay of the ESA probed at $1010 \mathrm{~nm}$ and the concomitant rise of the triplet at $730 \mathrm{~nm}$, a time constant of $378 \mathrm{ps}$ is assigned to the intersystem crossing (bottom inset of Figure 3a). We note that the fast decay at $730 \mathrm{~nm}$ is caused by the red-shift of overlapping SE and ESA features as 
discussed above. As elaborated in the Discussion, triplet formation is generally observed in disordered polythiophene systems where the singlet excited state is intrachain localized.

The early TA spectra at $0.5 \mathrm{ps}$ for CPT at four different temperatures $\left(5,25,40\right.$ and $\left.55^{\circ} \mathrm{C}\right)$ are compared in Figure 3b. They are dominated by the signatures of the singlet excited state. All the TA features (GSB, SE and ESA) at high temperature are blue-shifted compared to the ones at low temperature. Indeed, the GSB is gradually blue-shifting, becoming less structured, and the 00/0-1 ratio is decreasing with increasing temperature, which reflects what was discussed above for the stationary absorption spectra. The overlapping SE also blue-shifts and becomes structureless at high temperature. As mentioned above, mainly the $\mathrm{SE}$ is seen at $55^{\circ} \mathrm{C}$, the GSB being outside the spectral window. Finally, the broad positive ESA band is situated at $1050 \mathrm{~nm}$ at $5^{\circ} \mathrm{C}$ and progressively shifts towards higher energy with temperature to end with a sharper peak at $940 \mathrm{~nm}$ at $55^{\circ} \mathrm{C}$. The amplitude of the ESA and GSB bands is the highest at $5^{\circ} \mathrm{C}$, although the number of absorbed photons was kept constant. This indicates higher oscillator strength for those transitions in the more ordered conformations at low temperature, which we will discuss in terms of exciton delocalization. On the other hand, the $\mathrm{SE}$ at the lower temperatures (especially $25^{\circ} \mathrm{C}$ and $40^{\circ} \mathrm{C}$ ) is less pronounced compared to $55^{\circ} \mathrm{C}$. A similar reduction of the SE was observed in regioregular P3HT films compared to regiorandom ones, and is explained by interchain interactions (see Discussion). ${ }^{71}$ In Figure 3c, the spectra of CPT 1000 ps after excitation at 400 $\mathrm{nm}$ are compared for the different temperatures. Only the GSB and either the signature of the polarons (at low temperature) or of the triplet state (at high temperature) are visible, since the singlet excited state with the SE and ESA signatures has decayed at this long time scale. The GSB associated with the ordered phase that forms polarons $\left(\right.$ at $5-40^{\circ} \mathrm{C}$ ) has a similar amplitude at the different temperatures and varies only slightly in shape (stronger 0-0 band and slight red-shift at low temperature). The triplet state band is very intense for $55^{\circ} \mathrm{C}$, but also clearly visible at $40^{\circ} \mathrm{C}$ and $25^{\circ} \mathrm{C}$ (where it co-exists with the polaron signature), in contrast to the situation at $5^{\circ} \mathrm{C}$ where no peak is visible at $\sim 730 \mathrm{~nm}$. This shows that at the intermediate temperatures of $25^{\circ} \mathrm{C}$ and $40^{\circ} \mathrm{C}$, both the random-coil and ordered CPT conformations are populated at $400 \mathrm{~nm}$. Given that there is a mixture of conformations, there is inhomogeneity in the triplet state energy, and the triplet band shifts progressively from about $780 \mathrm{~nm}$ to $730 \mathrm{~nm}$ as the temperature is increased. On the other hand, the position of the polaron band is nearly the same between 5$40^{\circ} \mathrm{C}$. This is a further indication that polarons are formed only from the ordered phase, which 
shows less inhomogeneity. The polaron band seems slightly more intense at the higher temperatures, but this is only due to the superposition with the triplet state band.

\section{$580 \mathrm{~nm}$ excitation}
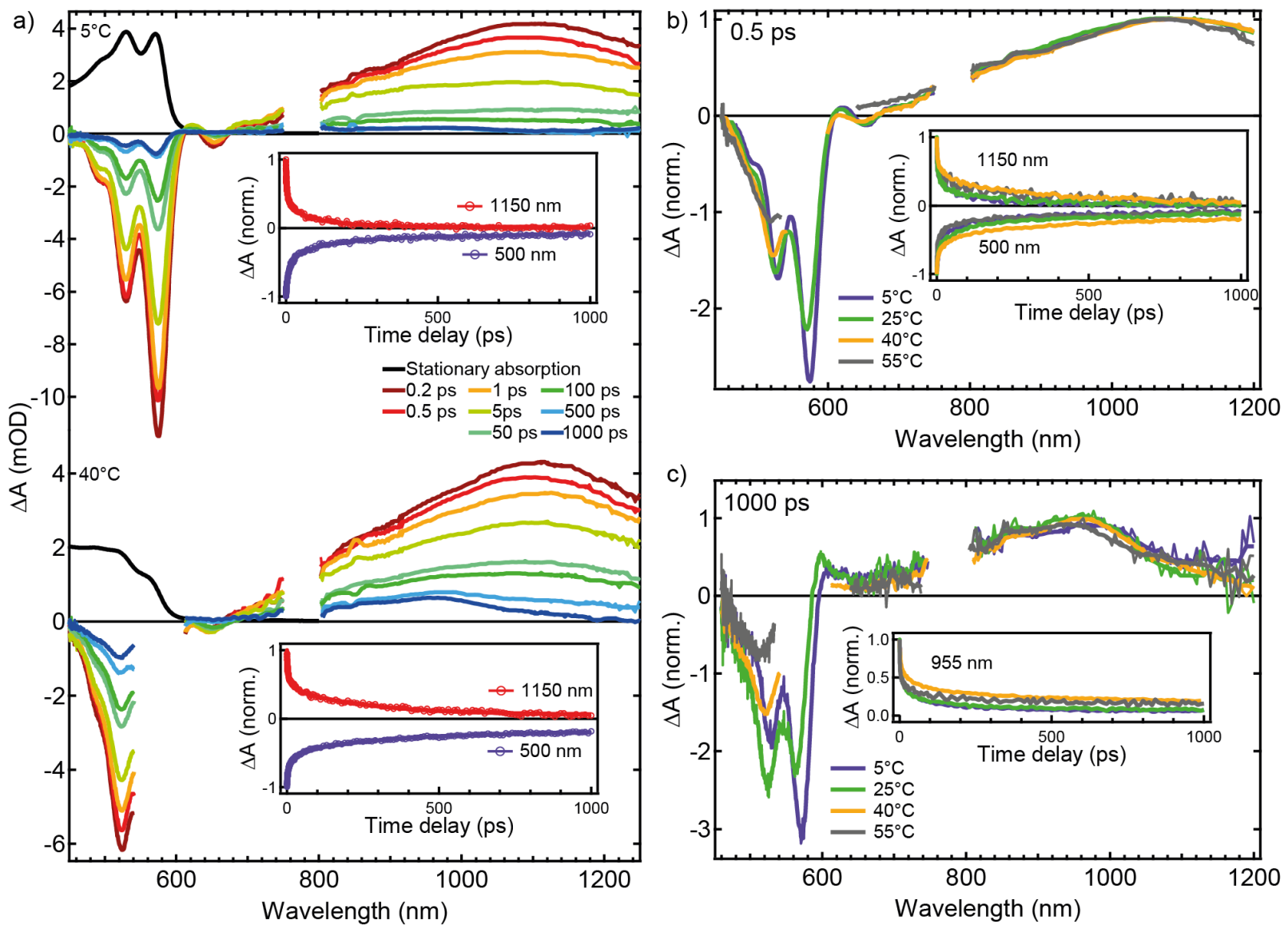

Figure 4. TA data for CPT in solution following excitation at $580 \mathrm{~nm}\left(1.5 \cdot 10^{-4} \mathrm{M}\right.$ on a monomeric unit basis in PBS buffer). (a) TA spectra recorded at selected time delays for CPT at $5^{\circ} \mathrm{C}$ (top) and $40^{\circ} \mathrm{C}$ (bottom) with the corresponding stationary absorption (black curves). The two insets show the TA dynamics probed at selected wavelengths. (b) Normalized TA spectra at four temperatures $\left(5,25,40\right.$ and $\left.55^{\circ} \mathrm{C}\right)$ at $0.5 \mathrm{ps}$ after photoexcitation. The inset shows the TA dynamics probed at 500 and $1150 \mathrm{~nm}$. (c) TA spectra at the four temperatures at $1000 \mathrm{ps}$ after photoexcitation. The inset shows the TA dynamics probed at $955 \mathrm{~nm}$. 
Figure 4a shows selected TA spectra for CPT in PBS buffer solution at $5^{\circ} \mathrm{C}$ and $40^{\circ} \mathrm{C}$ following excitation at $580 \mathrm{~nm}$. For both temperatures, this wavelength excites exclusively the more ordered phase of CPT, because it falls into the red side of the 0-0 vibronic absorption peak (see Figure 1b). According to the RR measurements, particularly planar chains are excited at higher wavelengths. To keep the number of absorbed photons constant in spite of weak absorption at the higher temperatures, a high excitation fluence was used at $40^{\circ} \mathrm{C}$, so that a portion of the spectrum around $580 \mathrm{~nm}$ is obscured by scattering of the pump. Since the fluence and scattering were even higher at $55^{\circ} \mathrm{C}$, this data is not shown here. The TA spectra of CPT at $5^{\circ} \mathrm{C}$ show a negative GSB feature below $600 \mathrm{~nm}$, with a well-defined vibronic progression at $574 \mathrm{~nm}, 530$ and $493 \mathrm{~nm}$. The 0-0/0-1 ratio in the GSB (1.8) is enhanced compared to the stationary absorption spectrum (0.98). Similarly to what was observed with $400 \mathrm{~nm}$ excitation at $5^{\circ} \mathrm{C}$, the signal at $574 \mathrm{~nm}$ can be partly ascribed to overlapping SE, together with the small indent at $653 \mathrm{~nm}$. The broad positive feature from $670 \mathrm{~nm}$ to $1250 \mathrm{~nm}$ observed at early times after photoexcitation is attributed to ESA from the singlet excited state. This excitonic band decays to zero with time constants of 0.7 ps, $9.4 \mathrm{ps}$ and $147 \mathrm{ps}$ (see dynamics at $1150 \mathrm{~nm}$ in the upper inset of Figure 4a). There is a concomitant complete decay of the SE and partial decay of the GSB, as shown in the dynamics at $500 \mathrm{~nm}$. At $1000 \mathrm{ps}$, only some GSB and a weak band (quite flat with a peak at $\sim 965 \mathrm{~nm}$ ) are visible. This band, resembling the one observed with $400 \mathrm{~nm}$ excitation, is ascribed to long-lived polarons. In contrast to excitation at $400 \mathrm{~nm}$, the TA spectra of CPT recorded with $580 \mathrm{~nm}$ excitation at $40^{\circ} \mathrm{C}$ are not significantly different from the low temperature case (bottom of Figure 4a), in agreement with the similar RR spectra excited at different temperatures at 473 $\mathrm{nm}$. As above, the structured negative band is due to overlapping GSB and SE, the broad positive band is due to ESA, and at long time delays, the features of the polarons are seen. The ESA band centred at $\sim 1100 \mathrm{~nm}$ is identical at all four investigated temperatures at $0.5 \mathrm{ps}$ (Figure $4 \mathrm{~b}$ ), and decays in a comparable way (inset of Figure $4 \mathrm{~b}$, at $1150 \mathrm{~nm}$ ). The TA spectra were normalized at $1100 \mathrm{~nm}$ for better comparison, since there was some uncertainty in their absolute amplitude due to the large range of fluences used to keep the number of absorbed photons constant. As seen for the data at $5^{\circ} \mathrm{C}$ and $40^{\circ} \mathrm{C}$ in Figure $4 \mathrm{a}$, the absolute amplitude at the different temperatures is nevertheless approximately similar. The comparison of the positive polaron absorption at 1000 ps in Figure 4c (normalized at $\sim 955 \mathrm{~nm}$ ) reveals no significant difference in the shape at the four temperatures, either. However, the amplitude of the polaron band at $40^{\circ} \mathrm{C}$ is higher than at $5^{\circ} \mathrm{C}$ 
(Figure 4a), which is also reflected in a long-lived offset in the dynamics at $955 \mathrm{~nm}$ and in the GSB at $500 \mathrm{~nm}$ (insets of Figure 4b,c). Although partly masked by scattering, there seems to be a decrease of the relative intensity of the $0-0$ vibronic peak in the GSB with increasing temperature, as in the stationary absorption spectra.

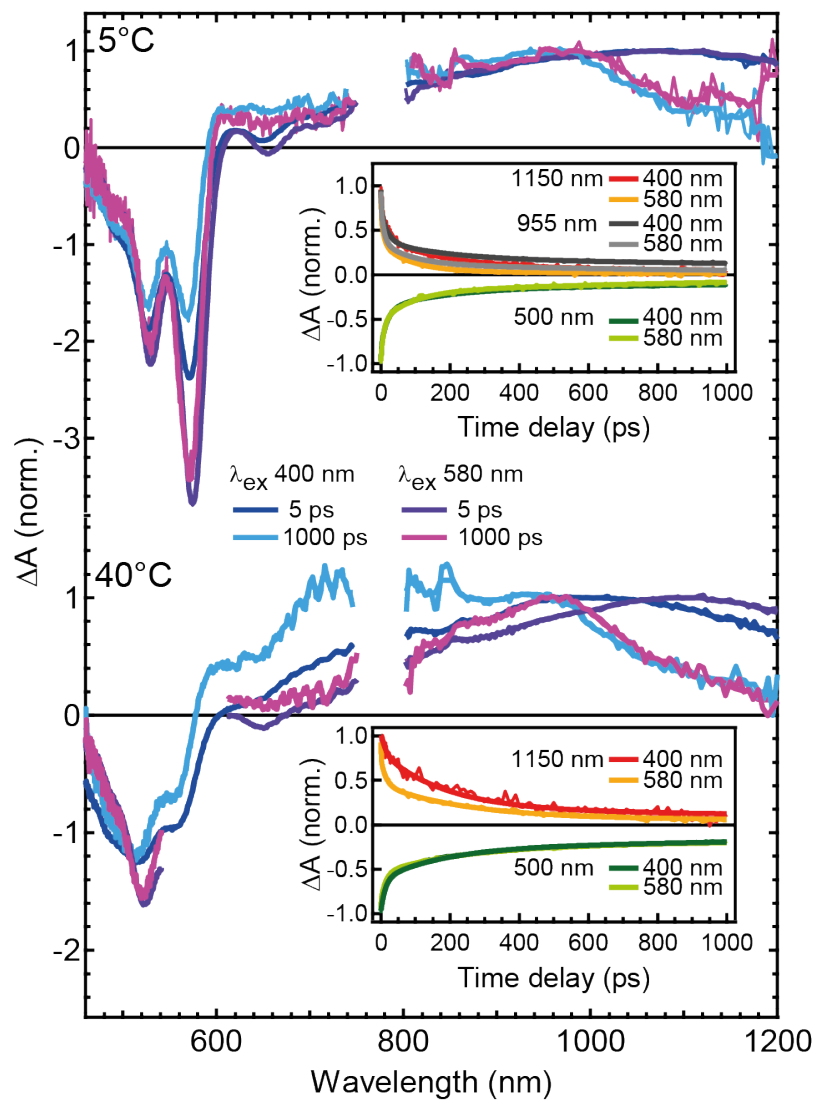

Figure 5. Normalized TA spectra for CPT at $5^{\circ} \mathrm{C}$ (top) and at $40^{\circ} \mathrm{C}$ (bottom) at 5 ps and 1000 ps after photoexcitation at $400 \mathrm{~nm}$ and $580 \mathrm{~nm}$. The two insets show the normalized TA dynamics probed at $1150 \mathrm{~nm}$ (ESA), $955 \mathrm{~nm}$ (ESA and polaron absorption) and at $500 \mathrm{~nm}$ (GSB).

Finally, we compare the behaviour of CPT in PBS when it is excited at the two excitation wavelengths of $400 \mathrm{~nm}$ and $580 \mathrm{~nm}$ (Figure 5, Figure $\mathrm{S} 10$ ). We have seen that at $5^{\circ} \mathrm{C}$, the ordered phase is populated in both cases (leading to polaron generation), but the RR measurements suggest higher backbone planarity with excitation at lower energy. Therefore, there are some differences in the TA spectra with the two excitation wavelengths, as shown in 
Figure 5a. At the early time delay of 5 ps, the GSB is narrower and slightly more intense at 580 $\mathrm{nm}$, with a larger 0-0/0-1 peak ratio, which partially also reflects an enhanced intensity of the SE. Moreover, the ESA at $580 \mathrm{~nm}$ is slightly red-shifted $(\sim 1100 \mathrm{~nm}$ versus $\sim 1050 \mathrm{~nm})$. Similar remarks can be made for the GSB and polaron features at 1000 ps. The inset of Figure 5a compares the normalized TA dynamics with the two excitations at various probe wavelengths, showing that they are overall very similar. Only the slightly larger long-lived offset at $955 \mathrm{~nm}$ indicates favoured polaron formation with higher excitation energy at $400 \mathrm{~nm}$. For CPT at $40^{\circ} \mathrm{C}$ (Figure 5b), more differences can be noticed between the two excitation wavelengths. Here, only the ordered phase is excited at $580 \mathrm{~nm}$, while both the random-coil and ordered phases are excited at $400 \mathrm{~nm}$. The signature of the GSB is in both cases dominated by the ordered phase, since the GSB of the random-coil conformation is outside the spectral window of the TA. Nevertheless, the GSB at $400 \mathrm{~nm}$ is much broader, reflecting the shape of the corresponding band in the stationary absorption spectrum of CPT at $40^{\circ} \mathrm{C}$ (Figure 1b), while the GSB at $580 \mathrm{~nm}$ is clearly much narrower. The corresponding ESA is also red-shifted to $\sim 1100 \mathrm{~nm}(580 \mathrm{~nm}$ excitation) compared to $\sim 1000 \mathrm{~nm}$ (400 nm excitation). At long time delays, one can observe how the position of the polaron absorption is almost unchanged between the two excitation wavelengths, while the presence of the triplet state is evident only at $400 \mathrm{~nm}$, where a mixture of random-coil and ordered conformations is excited. The dynamics at $500 \mathrm{~nm}$ (mainly GSB) are similar at the two excitation wavelengths, while the ESA at $1050 \mathrm{~nm}$ decays more slowly at 400 $\mathrm{nm}$. This is probably due to the contribution of intersystem crossing and spectral relaxation of the random-coil polymer chains excited at this wavelength.

\section{DISCUSSION}

Polythiophene photophysics. The photophysics of conjugated polymers and in particular polythiophenes has been thoroughly investigated. Experimental and theoretical studies have shown that the optical properties of polythiophenes are determined by a delicate balance of

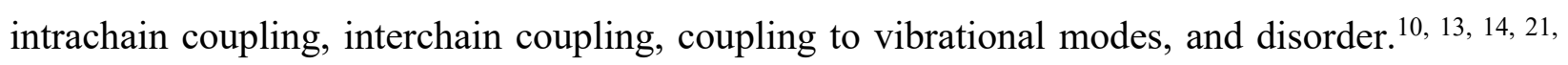
60,69 The intrachain coupling refers to the possibility of electron delocalisation along the polymer backbone and is thus closely related to the conjugation length. This is strongly linked to the chain conformation, i.e. the planarity between thiophene units (torsional order) and the overall 
chain extension. It has been shown that intrachain coupling leads to J-aggregate type behaviour in the optical transitions. ${ }^{10,12,73}$ The interchain coupling refers to the possibility of through-space delocalization across polymer chains that are aggregated or folded, and which are closely packed to allow molecular orbital overlap. This can be modelled as H-aggregate type behaviour. ${ }^{10,12,73}$ Again, good packing is favoured by order in the polymer backbone (planarity, extension), but also requires ordering of the side chains. Therefore, the regioregularity of the side chains plays an important role in determining the photophysics of polythiophenes, as has been shown for regioregular and regiorandom P3HT thin films and even single chains. ${ }^{15,67,74}$ In more ordered polythiophene systems, there is competition between inter- and intrachain coupling, and the overall photophysical properties are governed by subtle changes in the packing and chain conformation, which shift the system towards the H-aggregate or J-aggregate limit. ${ }^{12,73}$

Stationary absorption spectra allow distinguishing between the disordered phase of polythiophenes (blue-shifted, broad absorption band without vibronic structure) and the ordered phase (red-shifted absorption band with clear vibronic structure).$^{7-9}$ Moreover, the shape of the vibronic structure in the ordered phase gives information about the extent of inter- versus intramolecular coupling. For example in P3HT, the ordered chains in low-temperature solutions and thin films are typically described as weakly coupled H-aggregates, leading to suppression of the $0-0$ vibronic peak in the absorption spectrum and allowing the calculation of the interchain coupling from the $0-0$ to $0-1$ peak ratio. $., 10,13,16,17,19$ RR spectra show the vibrational modes that couple to the absorption transition and provide insight to the ground-state chain conformation, in particular about the planarity between thiophene units and thus the extent of torsional order. Finally, in TA spectroscopy, the properties of the excited state are explored. This includes the nature, delocalization and relaxation of excitons, as well as the population of triplet or polaron states. As discussed below, all those properties depend strongly on intramolecular backbone conformation, intermolecular packing and disorder. It must be noted however that nuclear relaxation occurs in the excited state away from the ground state geometry in the Franck-Condon region, and that local structural changes (leading amongst others to self-localization of the excitons) can occur even before the earliest measureable times of 100-200 fs. ${ }^{14,20,75}$

Salt effect on CPT. We have shown here for the CPT polyelectrolyte, that the presence of $\mathrm{Cl}^{-}$ anions in PBS buffer solution leads to increased order at room temperature compared to CPT in 
pure water, as can be seen by the appearance of the stationary absorption band related to the ordered polythiophene phase. The salt effect for a related cationic polythiophene (poly(3-alkoxy4-methylthiophene, PMNT)) has been investigated previously at comparable polymer concentration. ${ }^{63}$ It was found that anions, especially less hydrated (more hydrophobic) ones on the chaotrophic side of the Hofmeister series, affect the polymer conformation via two effects: First, by interacting with the cationic polymer side-chains and thus reducing electrostatic repulsion between the side chains, and second, by interacting directly with the backbone increasing its solubility and preventing hydrophobic collapse into a disordered structure. In the RR spectra measured for CPT in PBS solution at room temperature, we have observed significant changes when exciting the disordered phase at $405 \mathrm{~nm}$ or the ordered phase at 473 $\mathrm{nm}$. With the help of DFT calculations and based on existing literature, we could relate these changes to a decrease of $8-10^{\circ}$ of the average torsional angle between thiophene units in the ordered chains, i.e. to a decrease in torsional disorder. This is in line with previous work on the effect of salts on the conformation of a water-soluble cationic polythiophene, which shows a shift in the $\mathrm{C}_{\alpha}=\mathrm{C}_{\beta}$ band from 1493 to $1484 \mathrm{~cm}^{-1}$ upon going from water, to $\mathrm{KF}$, down the halogen group to $\mathrm{KI}$ and $\mathrm{KSCN} .{ }^{63}$ Molecular dynamics simulations indicated that in water and $\mathrm{KF}$, the polymer assumes a more random-coil conformation, while in $\mathrm{KI}$ and $\mathrm{KSCN}$ the polymer is more rod-like (ordered).

Ordered and disordered phase of CPT. The torsional and conformational disorder in the random coil phase of CPT leads to a broad distribution of relatively short conjugation lengths, explaining the broad blue-shifted absorption without vibronic structure that we have observed in pure water or in PBS at the higher measured temperatures. In the TA data with excitation of the disordered conformations, this inhomogeneity is further evidenced by the gradual red shift of the SE and ESA features due to excitation energy transfer from shorter to the longer polymer segments. We have not seen this effect when exciting the ordered phase, showing that it does not only have a more planar backbone, but also a more homogeneous distribution of conjugation lengths. The higher homogeneity of the ordered phase is confirmed by the presence of the vibronic progression in the corresponding stationary absorption band, ${ }^{14,71}$ and by the narrower bands in the RR spectrum excited at $473 \mathrm{~nm}$. Our TA measurements have shown further differences in the excited-state behaviour of the disordered and ordered phase of CPT. While only excitons are directly generated in the disordered conformations, both excitons and polarons 
appear within the time resolution of the experiment for the ordered phase (vide infra). Moreover, the ESA in the near infrared region is systematically more blue-shifted and has lower oscillator strength for the disordered chains. This can be explained by the shorter conjugation length in the random-coil phase, leading to more intrachain localized excitons with higher energy, in contrast to the more (intrachain and/or interchain) delocalized excitons with lower energy of the ordered phase. The same tendency has been previously observed by Cook et al. when comparing P3HT in solution (random-coil) versus in thin films (ordered), where the ESA band shifted from 1000 to $\sim 1240 \mathrm{~nm},{ }^{18}$ or by Ito et al. who compared regiorandom P3HT films (amorphous) with regioregular P3HT films (semi-crystalline) and observed a shift of the ESA from 1000 to 1200 nm. ${ }^{15}$ We have also observed that intersystem crossing to the triplet state occurs only in the random-coil conformations. In contrast, the excitons in the ordered phase decay to the ground state. It has been demonstrated with quantum-chemical calculations that spin-orbit coupling, which favours triplet state formation, increases with increasing dihedral angle between the thiophene rings for oligothiophenes. ${ }^{72,} 76$ Since we have shown by RR spectroscopy that the average torsional angle between thiophene units in CPT is higher in the random coil phase, triplet formation is indeed favoured for the disordered conformations. The triplet state was also identified by Cook et al. at $870 \mathrm{~nm}$ for P3HT in room-temperature solution. ${ }^{18}$ In the solid state, the long-lived triplet state was identified in regiorandom P3HT films, which are known to be less ordered than regioregular P3HT. ${ }^{15,71}$

Thermochromism of CPT. We have controlled the ratio between the disordered and ordered phase of CPT in PBS solution by changing the temperature. As discussed below, the temperature also affects the extent of inter- versus intrachain coupling in the ordered phase. A qualitatively similar temperature-dependence to CPT in aqueous PBS solution has been reported for the absorption spectrum of P3HT in tetrahydrofuran and methylcyclohexane. ${ }^{9}, 11$ The conversion from random-coil to ordered chains typically takes place at lower temperature $\left(<-10^{\circ} \mathrm{C}\right)$ than what we observe for CPT in PBS, and over a narrower temperature range. It was described as a first order disorder-to-order phase transition, whereby the transition temperature depends on the polymer concentration and molecular weight. Therefore, a broad range in transition temperature is typical for polymers with high polydispersity (for example CPT, where PDI = 2.0). ${ }^{9}$ It was reported for P3HT that when decreasing the temperature, the polymer chains first planarize, then extend and finally collapse into ordered aggregates upon ordering of the side chains. For CPT, 
we have shown by RR and TA spectroscopy that the ordered phase at low temperature consists of more planar chains with a more homogeneous distribution of conjugation lengths. This situation could in principle be due to isolated extended chains that are stabilized by the ions present in the dilute PBS solution. Nevertheless, we show in the following that, similar to the P3HT solutions at low temperature, interchain packing also occurs in the ordered phase of CPT. Although we did not notice any concentration dependence within the investigated range in the absorption spectra of CPT at different temperatures (Figure S1), dynamic light-scattering measurements revealed that particles with a diameter up to $120-150 \mathrm{~nm}$ persist even in the most dilute and ultrasonicated solutions at room temperature (Table S1, Figure S11). This is consistent with a report revealing supramolecular assemblies in aqueous CPT solutions. ${ }^{53}$ We note that those extremely large clusters, which we will refer to as loose agglomerates, do not necessarily have any spectroscopic significance, since they do not imply any close packing of the polymer backbones. Loose agglomerates with random-coil chain conformations also exist for P3HT at low concentration in good organic solvents. ${ }^{77}$

Although the agglomerates of CPT in PBS are not necessarily associated with order, their presence implies that the CPT chains are not isolated, so that they can potentially form interchain aggregates when the backbones planarize in the ordered phase, especially since the electrostatic repulsion of the cationic side-chains is reduced at the high salt concentration. In the stationary absorption spectrum at room temperature, we have seen indeed a suppressed 0-0 band for CPT in PBS buffer, confirming the presence of H-aggregates in the ordered phase. However, we have also observed an increase of the relative 0-0 intensity as the temperature was further decreased below room temperature. At $1^{\circ} \mathrm{C}$, the $0-0$ to $0-1$ peak ratio is close to 1 , showing that the polymer chains are no longer in the H-aggregate limit. This is in clear contrast to the reported results for P3HT in tetrahydrofuran, where there was no pronounced change in the 0-0 to 0-1 peak ratio and the relative intensity of the $0-0$ peak remained suppressed at the lowest measured temperatures. ${ }^{9}$ For CPT in PBS buffer, our results thus show that below $10^{\circ} \mathrm{C}$, the ordered phase has predominant J-like behaviour, with a subsequent increase of the 0-0 vibronic band, and reduced interchain coupling. A similar J-aggregate behaviour has been observed in highly ordered P3HT nanofibres. ${ }^{12}$ It points to significant backbone extension in the ordered phase of CPT at low temperature. The remaining electrostatic repulsion between the charged side-chains helps to reduce the relative contribution of interchain coupling and to shift the system towards the J- 
aggregate limit. Also, the regioregularity of CPT is not as high as in typical regioregular P3HT, which reduces the interchain packing. ${ }^{78}$

Intermolecular vs. intramolecular coupling. The extent of inter- versus intramolecular interactions could be confirmed by TA spectroscopy and correlated to the $0-0$ to $0-1$ peak ratio of the stationary absorption spectra. The RR spectra with excitation of the ordered phase of CPT at $473 \mathrm{~nm}$ did however not show any significant temperature dependence, showing that very weak changes in torsional angle are sufficient to affect the extent of intermolecular coupling. For the TA, we have found that a higher $\mathrm{H}$-aggregate character of the ordered phase leads to polaron formation and reduced stimulated emission. Various mechanisms for the generation of polarons in neat polythiophene or other conjugated polymer films have been suggested, including intrinsic free charge generation, ${ }^{79}$ dissociation of excitons at defects, ${ }^{80}$ at ordered/amorphous interfaces, ${ }^{81}$ ultrafast hot exciton dissociation, ${ }^{15}$ or exciton-exciton annihilation. ${ }^{82,83}$ For CPT, we can exclude annihilation based on the absence of any fluence-dependence in the TA dynamics and polaron yield (Figure S12). Given that there is no sign of a slowly rising polaron signature in the TA data, we can also conclude that the polarons are formed within the time resolution of the experiment ( $<200 \mathrm{fs})$ and co-exist with a separate population of excitons. ${ }^{18}$ The generation of long-lived polarons in P3HT is typically linked to the presence of the ordered phase in regioregular thin films, and almost no polarons are observed for P3HT dissolved in good solvents or in regiorandom $\mathrm{P} 3 \mathrm{HT}$ films, where random-coil polymer conformations are adopted. ${ }^{15,18}$ We therefore directly relate the formation of polarons for CPT in PBS buffer to the excitation of the ordered phase. Either J-type intrachain delocalization along the ordered backbone could lead to charges within the same polymer chain, or charges could be generated across neighbouring backbones thanks to interchain H-aggregation. ${ }^{73}$

Judging from previous studies with P3HT nanofibres and thin films, ${ }^{73}, 84$ the polaron yield is generally much higher in the latter case, providing evidence that aggregation of CPT in the ordered phase enhances the observed polaron generation. In line with this we have observed that, with excitation of the ordered phase at $580 \mathrm{~nm}$, polaron formation is slightly favoured at higher temperature $\left(40^{\circ} \mathrm{C}\right.$ compared to $\left.5^{\circ} \mathrm{C}\right)$, where the stationary absorption spectra with reduced relative 0-0 intensity suggest that the interchain $\mathrm{H}$-aggregate character is enhanced. When exciting at $400 \mathrm{~nm}$ (where the ordered phase is increasingly excited at lower temperature), we 
have also seen a reduction of the $\mathrm{SE}$ at the intermediate temperatures $\left(40^{\circ} \mathrm{C}\right.$ and $\left.25^{\circ} \mathrm{C}\right)$, where the ordered phase is more H-type, making the 0-0 emission forbidden. The more pronounced SE at $5^{\circ} \mathrm{C}$ confirms a larger contribution of intrachain J-coupling at the lowest measured temperature. At this temperature, the polaron yield (seen as the long-lived offset in the TA data) is higher with $400 \mathrm{~nm}$ excitation than with $580 \mathrm{~nm}$ excitation. This agrees with the narrower GSB features, the enhanced SE and the red-shifted ESA at $580 \mathrm{~nm}$, which all point to excitation of more extended CPT chains having a higher degree of intrachain delocalization and J-aggregate behaviour, and thus weaker polaron yield. In the RR spectra, the higher degree of torsional order with excitation at $473 \mathrm{~nm}$ compared to $405 \mathrm{~nm}$ at $5^{\circ} \mathrm{C}$ is even more pronounced. Indeed at $405 \mathrm{~nm}$, the temperature dependence of the band intensity points to a decrease of the average torsional angle between the thiophenes by about $10^{\circ}$ at $5^{\circ} \mathrm{C}$. This is enough to significantly affect the excitedstate behaviour in the TA data, promoting interchain packing ( $\mathrm{H}$-aggregate character) and polaron formation. We note that there might also be additional backbone planarization in the excited state compared to the ground state, affecting the TA but not RR measurements. Overall, we conclude that there remains a small spread of conformations even in the more homogeneous ordered phase of CPT. When exciting at $400 \mathrm{~nm}$, the ensemble of more ordered conformations contributes to the TA features at the lowest temperatures, while at $580 \mathrm{~nm}$ only a subset of the most extended chains is excited.

\section{CONCLUSION}

We have investigated the temperature-dependent stationary absorption, resonance Raman and femtosecond-resolved transient absorption spectra of a conjugated polythiophene with cationic side-chains, dissolved in an aqueous biological environment consisting of phosphate buffered saline (PBS) solution. We have found that the high concentration of chlorine anions $\left(\mathrm{Cl}^{-}\right)$ contained in PBS favours backbone planarization of the polyelectrolyte, leading to the observation of an ordered phase even at room temperature. In pure water or in PBS solution at high temperature $\left(>50^{\circ} \mathrm{C}\right)$, more disordered and inhomogeneous random-coil conformations are predominant. The ordered phase shows both $\mathrm{H}$-aggregate behaviour due to interchain interactions, and J-aggregate behaviour due to intrachain coupling along the backbone. At the lowest measured temperature $\left(1^{\circ} \mathrm{C}\right)$, the J-type behaviour dominates due to enhanced chain extension, and the $0-0$ vibronic absorption band is no longer suppressed. We demonstrate that 
photoexcitation of the ordered phase leads to the ultrafast generation of long-lived polarons, similar to what has been reported for regiorandom P3HT thin films. This is assisted by interchain aggregation. On the other hand, increased torsional disorder in the random-coil conformations at high temperature prevents the formation of polarons, and instead there is relatively slow ( $\sim 400$ ps) intersystem crossing to the triplet state. Conjugated polyelectrolytes are commonly used in biological environments to detect macromolecules such as DNA. In order to understand and to optimize the sensing strategies, it is essential to first characterize the photophysical behaviour and its dependence on the polyelectrolyte conformation in the biological solution without the added macromolecules. As a next step, we are currently investigating how assembly of the cationic polythiophene with single DNA strands modifies its conformation, leading to rich optical sensing possibilities.

\section{ACKNOWLEDGEMENTS}

N.B., L.P. and Y.S. acknowledge the Swiss National Science Foundation (grant PP00P2_150536) and the University of Fribourg for financial support. This project has received funding from the European Union's Horizon 2020 research and innovation programme under the Marie Sklodowska-Curie grant agreements No 643238. We thank Prof. Carlos Silva (Georgia Tech) and Prof. Epameinondas Leontidis (Univ. of Cyprus) for fruitful discussions.

1. A. Facchetti, Chem. Mater., 2011, 23, 733-758.

2. A. J. Heeger, N. S. Sariciftci and E. B. Namdas, Semiconducting and metallic polymers, Oxford University Press, Oxford, UK, 2010.

3. B. C. Thompson and J. M. J. Fréchet, Angew. Chem., Int. Ed., 2008, 47, 58-77.

4. Z. L. Li, S. C. Yang, H. F. Meng, Y. S. Chen, Y. Z. Yang, C. H. Liu, S. F. Horng, C. S. Hsu, L. C. Chen, J. P. Hu and R. H. Lee, Appl. Phys. Lett., 2004, 84, 3558-3560.

5. Y. Kim, S. Cook, S. M. Tuladhar, S. A. Choulis, J. Nelson, J. R. Durrant, D. D. C. Bradley, M. Giles, I. McCulloch, C.-S. Ha and M. Ree, Nat. Mater., 2006, 5, 197-203.

6. S. Günes, H. Neugebauer and N. S. Sariciftci, Chem. Rev., 2007, 107, 1324-1338.

7. Y. D. Park, H. S. Lee, Y. J. Choi, D. Kwak, J. H. Cho, S. Lee and K. Cho, Adv. Funct. Mater., 2009, 19, 1200-1206. 
8. F. Panzer, M. Sommer, H. Bässler, M. Thelakkat and A. Köhler, Macromolecules, 2015, 48, 1543-1553.

9. F. Panzer, H. Bässler, R. Lohwasser, M. Thelakkat and A. Köhler, J. Phys. Chem. Lett., 2014, 5, 2742-2747.

10. F. C. Spano and C. Silva, Annu. Rev. Phys. Chem., 2014, 65, 477-500.

11. B. Ferreira, P. F. da Silva, J. S. Seixas de Melo, J. Pina and A. Maçanita, J. Phys. Chem. $B, 2012,116,2347-2355$.

12. E. T. Niles, J. D. Roehling, H. Yamagata, A. J. Wise, F. C. Spano, A. J. Moulé and J. K. Grey, J. Phys. Chem. Lett., 2012, 3, 259-263.

13. H. Yamagata and F. C. Spano, J. Chem. Phys., 2012, 136, 184901.

14. N. Banerji, S. Cowan, E. Vauthey and A. J. Heeger, J. Phys. Chem. C, 2011, 115, 97269739.

15. J. M. Guo, H. Ohkita, H. Benten and S. Ito, J. Am. Chem. Soc., 2009, 131, 16869-16880.

16. J. Clark, J.-F. Chang, F. C. Spano, R. H. Friend and C. Silva, Appl. Phys. Lett., 2009, 94, 163306.

17. F. C. Spano, J. Clark, C. Silva and R. H. Friend, J. Chem. Phys., 2009, 130, 074904.

18. S. Cook, A. Furube and R. Katoh, Energy Environ. Sci., 2008, 1, 294-299.

19. J. Clark, C. Silva, R. H. Friend and F. C. Spano, Phys. Rev. Lett., 2007, 98, 206406.

20. N. P. Wells, B. W. Boudouris, M. A. Hillmyer and D. A. Blank, J. Phys. Chem. C, 2007, 111, 15404-15414.

21. P. J. Brown, D. S. Thomas, A. Köhler, J. S. Wilson, J. S. Kim, C. M. Ramsdale, H. Sirringhaus and R. H. Friend, Phys. Rev. B, 2003, 67, 064203.

22. X. M. Jiang, R. Österbacka, O. Korovyanko, C. P. An, B. Horovitz, R. A. J. Janssen and Z. V. Vardeny, Adv. Funct. Mater., 2002, 12, 587-597.

23. A. Duarte, K.-Y. Pu, B. Liu and G. C. Bazan, Chem. Mater., 2011, 23, 501-515.

24. T. Yamamoto, D. Komarudin, M. Arai, B.-L. Lee, H. Suganuma, N. Asakawa, Y. Inoue, K. Kubota, S. Sasaki, T. Fukuda and H. Matsuda, J. Am. Chem. Soc., 1998, 120, 20472058.

25. H. Choi, C.-K. Mai, H.-B. Kim, J. Jeong, S. Song, G. C. Bazan, J. Y. Kim and A. J. Heeger, Nat. Commun., 2015, 6, 7348.

26. H. Yan, C. Catania and G. C. Bazan, Adv. Mater., 2015, 27, 2958-2973. 
27. X. F. Liu, Q. L. Fan and W. Huang, Biosens. Bioelectron., 2011, 26, 2154-2164.

28. Y. Liu, K. Ogawa and K. S. Schanze, J. Photochem. Photobiol., C, 2009, 10, 173-190.

29. G. C. Bazan and S. Wang, Springer Ser. Mater. Sci., 2008, 107, 1-37.

30. F. Wang, M. Li, B. Wang, J. Zhang, Y. Cheng, L. Liu, F. Lv and S. Wang, Sci. Rep., $2015,5,7617$.

31. K. Lee, J. Lee, E. J. Jeong, A. Kronk, K. S. Elenitoba-Johnson, M. S. Lim and J. Kim, Adv. Mater., 2012, 24, 2479-2484.

32. Y. Huang, C. Song, H. Li, R. Zhang, R. Jiang, X. Liu, G. Zhang, Q. Fan, L. Wang and W. Huang, ACS Appl. Mater. Interfaces, 2015, 7, 21529-21537.

33. G. Feng, C.-K. Mai, R. Zhan, G. C. Bazan and B. Liu, J. Mater. Chem. B, 2015, 3, 73407346.

34. H. Yuan, B. Wang, F. Lv, L. Liu and S. Wang, Adv. Mater., 2014, 26, 6978-6982.

35. Y. Wang, K. S. Schanze, E. Y. Chi and D. G. Whitten, Langmuir, 2013, 29, $10635-$ 10647.

36. J. M. Hodgkiss, G. Tu, S. Albert-Seifried, W. T. S. Huck and R. H. Friend, J. Am. Chem. Soc., 2009, 131, 8913-8921.

37. H. Choi, C.-K. Mai, H.-B. Kim, J. Jeong, S. Song, G. C. Bazan, J. Y. Kim and A. J. Heeger, 2015, 6, 7348.

38. E. Ji, A. Parthasarathy, T. S. Corbitt, K. S. Schanze and D. G. Whitten, Langmuir, 2011, 27, 10763-10769.

39. R. C. Huber, A. S. Ferreira, R. Thompson, D. Kilbride, N. S. Knutson, L. S. Devi, D. B. Toso, J. R. Challa, Z. H. Zhou, Y. Rubin, B. J. Schwartz and S. H. Tolbert, Science, 2015, 348, 1340.

40. I. Charlebois, C. Gravel, N. Arrad, M. Boissinot, M. G. Bergeron and M. Leclerc, Macromol. Biosci., 2013, 13, 717-722.

41. H.-A. Ho, M. Bera-Aberem and M. Leclerc, Chem. Eur. J., 2005, 11, 1718-1724.

42. H.-A. Ho and M. Leclerc, J. Am. Chem. Soc., 2004, 126, 1384-1387.

43. K. Dore, S. Dubus, H. A. Ho, I. Levesque, M. Brunette, G. Corbeil, M. Boissinot, G. Boivin, M. G. Bergeron, D. Boudreau and M. Leclerc, J. Am. Chem. Soc., 2004, 126, 4240-4244. 
44. H. A. Ho, M. Boissinot, M. G. Bergeron, G. Corbeil, K. Dore, D. Boudreau and M. Leclerc, Angew. Chem., Int. Ed., 2002, 41, 1548-1551.

45. N. Le Bouch, M. Auger and M. Leclerc, Macromol. Chem. Phys., 2008, 209, 2455-2462.

46. A. Köhler, S. T. Hoffmann and H. Bässler, J. Am. Chem. Soc., 2012, 134, 11594-11601.

47. C.-C. Wang, Y. Gao, A. P. Shreve, C. Zhong, L. Wang, K. Mudalige, H.-L. Wang and M. Cotlet, J. Phys. Chem. B, 2009, 113, 16110-16117.

48. G. H. Darwish, J. Abouzeid and P. Karam, RSC Advances, 2016, 6, 67002-67010.

49. G. H. Darwish, A. Koubeissi, T. Shoker, S. Abou Shaheen and P. Karam, Chem. Commun., 2016, 52, 823-826.

50. A. D. Becke, J. Chem. Phys., 1993, 98, 5648-5652.

51. T. Yanai, D. P. Tew and N. C. Handy, Chem. Phys. Lett., 2004, 393, 51-57.

52. M. J. Frisch, G. W. Trucks, H. B. Schlegel, G. E. Scuseria, M. A. Robb, J. R. Cheeseman, G. Scalmani, V. Barone, B. Mennucci, G. A. Petersson and e. al., 2009, Gaussian Inc., Wallingford CT.

53. P. Urbánek, A. di Martino, S. Gladyš, I. Kuřitka, A. Minařík, E. Pavlova and D. Bondarev, Synth. Met., 2015, 202, 16-24.

54. G. Zerbi, B. Chierichetti and O. Ingänas, J. Chem. Phys., 1991, 94, 4646-4658.

55. G. Louarn, J. P. Buisson, S. Lefrant and D. Fichou, J. Phys. Chem., 1995, 99, 1139911404.

56. A. Pron, G. Louarn, M. Lapkowski, M. Zagorska, J. Glowczy-Zubek and S. Lefrant, Macromolecules, 1995, 28, 4644-4649.

57. G. Louarn, M. Trznadel, J. P. Buisson, J. Laska, A. Pron, M. Lapkowski and S. Lefrant, J. Phys. Chem., 1996, 100, 12532-12539.

58. M. Baibarac, M. Lapkowski, A. Pron, S. Lefrant and I. Baltog, J. Raman Spectrosc., 1998, 29, 825-832.

59. Y. Gao, T. P. Martin, E. T. Niles, A. J. Wise, A. K. Thomas and J. K. Grey, J. Phys. Chem. C, 2010, 114, 15121-15128.

60. W. C. Tsoi, D. T. James, J. S. Kim, P. G. Nicholson, C. E. Murphy, D. D. C. Bradley, J. Nelson and J.-S. Kim, J. Am. Chem. Soc., 2011, 133, 9834-9843.

61. G. Shi, J. Xu and M. Fu, J. Phys. Chem. B, 2002, 106, 288-292.

62. F. e. Chen, G. Shi, J. Zhang and M. Fu, Thin Solid Films, 2003, 424, 283-290. 
63. M. Qiu, S. Long, B. Li, L. Yan, W. Xie, Y. Niu, X. Wang, Q. Guo and A. Xia, J. Phys. Chem. C, 2013, 117, 21870-21878.

64. Y. Gao and J. K. Grey, J. Am. Chem. Soc., 2009, 131, 9654-9662.

65. A. P. Scott and L. Radom, J. Phys. Chem., 1996, 100, 16502-16513.

66. N. Banerji, S. Cowan, M. Leclerc, E. Vauthey and A. J. Heeger, J. Am. Chem. Soc., 2010, 132, 17459-17470.

67. J. M. Guo, H. Ohkita, H. Benten and S. Ito, J. Am. Chem. Soc., 2010, 132, 6154-6164.

68. I. A. Howard, R. Mauer, M. Meister and F. Laquai, J. Am. Chem. Soc., 2010, 132, 1486614876.

69. W. Zhang, R. Hu, D. Li, M.-M. Huo, X.-C. Ai and J.-P. Zhang, J. Phys. Chem. C, 2012, 116, 4298-4310.

70. S. Westenhoff, W. J. D. Beenken, R. H. Friend, N. C. Greenham, A. Yartsev and V. Sundström, Phys. Rev. Lett., 2006, 97, 166804.

71. O. J. Korovyanko, R. Österbacka, X. M. Jiang, Z. V. Vardeny and R. A. J. Janssen, Phys. Rev. B, 2001, 64, 235122 .

72. H. Ohkita, S. Cook, Y. Astuti, W. Duffy, S. Tierney, W. Zhang, M. Heeney, I. McCulloch, J. Nelson, D. D. C. Bradley and J. R. Durrant, J. Am. Chem. Soc., 2008, 130, 3030-3042.

73. T. P. Martin, A. J. Wise, E. Busby, J. Gao, J. D. Roehling, M. J. Ford, D. S. Larsen, A. J. Moulé and J. K. Grey, J. Phys. Chem. B, 2013, 117, 4478-4487.

74. T. Adachi, J. Brazard, R. J. Ono, B. Hanson, M. C. Traub, Z.-Q. Wu, Z. Li, J. C. Bolinger, V. Ganesan, C. W. Bielawski, D. A. Vanden Bout and P. F. Barbara, J. Phys. Chem. Lett., 2011, 2, 1400-1404.

75. T. E. Dykstra, V. Kovalevskij, X. J. Yang and G. D. Scholes, Chem. Phys., 2005, 318, 21-32.

76. D. Beljonne, Z. Shuai, G. Pourtois and J. L. Bredas, J. Phys. Chem. A, 2001, 105, 38993907.

77. D. Raithel, S. Baderschneider, T. B. de Queiroz, R. Lohwasser, J. Kohler, M. Thelakkat, S. Kummel and R. Hildner, Macromolecules, 2016, 49, 9553-9560.

78. D. Bondarev, J. Zedník, I. Šloufová, A. Sharf, M. Procházka, J. Pfleger and J. Vohlídal, J. Polym. Sci. A Polym. Chem., 2010, 48, 3073-3081. 
79. P. B. Miranda, D. Moses and A. J. Heeger, Phys. Rev. B, 2001, 6408, 081201.

80. C. Silva, M. A. Stevens, D. M. Russell, S. Setayesh, K. Mullen and R. H. Friend, Synth. Met., 2001, 116, 9-13.

81. F. Paquin, G. Latini, M. Sakowicz, P. L. Karsenti, L. J. Wang, D. Beljonne, N. Stingelin and C. Silva, Phys. Rev. Lett., 2011, 106, 197401.

82. M. A. Stevens, C. Silva, D. M. Russell and R. H. Friend, Phys. Rev. B, 2001, 63, 165213.

83. D. Peckus, A. Devizis, D. Hertel, K. Meerholz and V. Gulbinas, Chem. Phys., 2012, 404, 42-47.

84. C. X. Sheng, M. Tong, S. Singh and Z. V. Vardeny, Phys. Rev. B, 2007, 75, 085206. 